\title{
Ferroelectricity from iron valence ordering in rare earth ferrites?
}

\author{
Manuel Angst ${ }^{*}, 1,2$ \\ ${ }^{1}$ Peter Grünberg Institut PGI and Jülich Centre for Neutron Science JCNS, JARA-FIT, Forschungszentrum Jülich GmbH, 52425 Jülich, \\ Germany \\ ${ }^{2}$ Experimental Physics IVC, RWTH Aachen University, 52056 Aachen, Germany
}

Received XXXX, revised XXXX, accepted XXXX

Published online 19 April 2013 - Phys. Status Solidi RRL, DOI:10.1002/pssr.201307103 (2013)

Key words: Multiferroics, Ferroelectrics, Charge Order, Spin-Charge Coupling, LuFe2O4, Rare Earth Ferrites

* Corresponding author: e-mail m.angst@fz-juelich.de, Phone: +49-2461-612479

The possibility of multiferroicity arising from charge ordering in $\mathrm{LuFe}_{2} \mathrm{O}_{4}$ and structurally related rare earth ferrites is reviewed. Recent experimental work on macroscopic indications of ferroelectricity and microscopic determination of coupled spin and charge order indicates that this scenario does not hold. Understanding the origin of the experimentally observed charge and spin order will require further theoretical work. Other aspects of recent research in these materials, such as geometrical frustration effects, possible electric-field-induced transitions, or orbital order are also briefly treated.

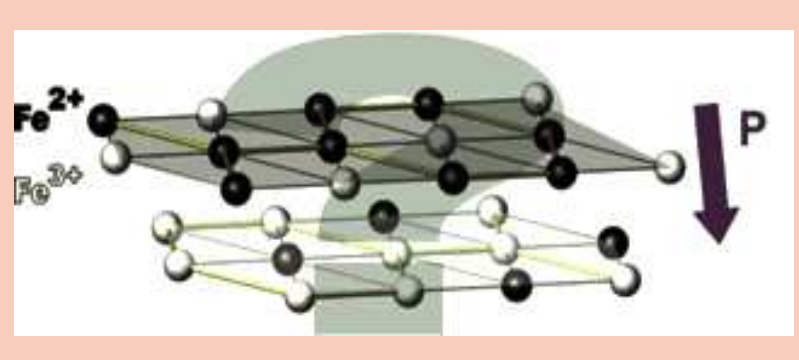

Bilayer subunit in $\mathrm{RFe}_{2} \mathrm{O}_{4}$ compounds with proposed charge order rendering it polar.

Copyright line will be provided by the publisher

1 Introduction The interplay of magnetic and electric degrees of freedom in correlated electron materials is one of the major topics of contemporary condensed matter physics, with a rapidly rising number of publications per year [1]. Materials combining magnetism with ferroelectricity, termed multiferroics, offer the prospect of a large magnetoelectric coupling, which has a high potential for applications in future information technology [2]. Because the traditional mechanism of ferroelectricity is incompatible with magnetism [3], much interest has focused on unconventional routes to ferroelectricity (see [4] for a recent extensive review). Particularly intriguing is the mechanism of ferroelectricity originating from charge ordering $(\mathrm{CO})$ (reviewed in [5,6]), the ordered arrangement of different valence states of an ion, typically a transition metal. Because any ferroelectric polarization is built from electric dipole moments, i.e. non-centrosymmetric charge distributions, any $\mathrm{CO}$ breaking inversion symmetry automatically induces a polarization, which may be very large [5]. The presence of different valence states of a transition metal ion implies an active spin degree of freedom on the same ion, and therefore a strong magnetoelectric coupling may be expected as well. This mechanism of multiferroicity is thus very attractive from the point of view of prospective applications. However, while the concept is straight-forward, examples of oxides where this mechanism is experimentally indicated to occur are exceedingly rare and none is really well understood.

The often cited (e.g. [4,5,6]) prototypical example material providing "proof of principle" for this mechanism is $\mathrm{LuFe}_{2} \mathrm{O}_{4}$, a charge- (and spin-) frustrated system. In the following, I will review recent research on this and isostructural materials, which may serve also as an illuminating case study for (much less investigated) other potential examples of CO-based ferroelectricity. $\mathrm{LuFe}_{2} \mathrm{O}_{4}$ had been proposed in 2005 to be a multiferroic due to $\mathrm{Fe}^{2+/ 3+}$ $\mathrm{CO}$ [17], based on both macroscopic indications by dielectric spectroscopy and pyroelectric current measurements, 


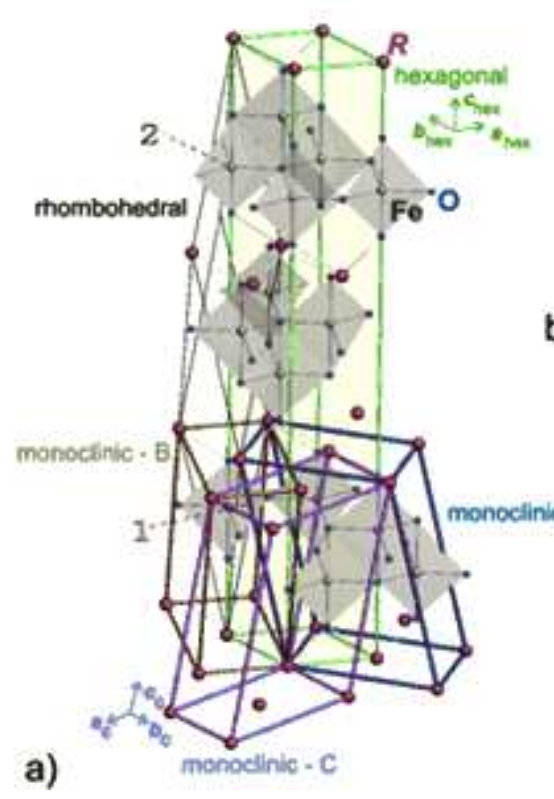

c)

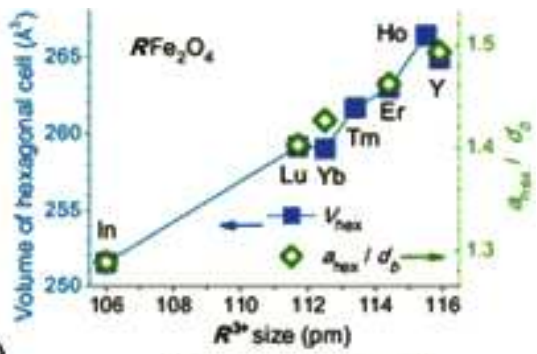

b)

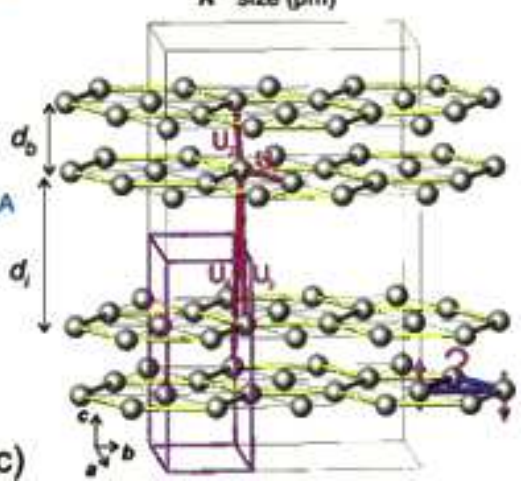

Figure 1 a) $R \overline{3} m$ Crystal structure of $R \mathrm{Fe}_{2} \mathrm{O}_{4}$ and description with different cells (after [7]). Apart from the primitive rhombohedral and the $R$-centered hexagonal cells the lattice could also be described by one of three $C$-centered monoclinic cells, which are rotated by $120^{\circ}$ with respect to each other. Cells describing the charge and spin order in the three domains are obtained from these by tripling the monoclinic $b$ and doubling the monoclinic $c$ axes (omitted for clarity). b) $R^{3+}$ ion size effect on cell volume and ratio of intralayer $\mathrm{Fe}-\mathrm{Fe}$ distance $a_{\text {hex }}$ to bilayer thickness $d_{b}$ (c.f. c), compiled from [89, 10, 11, 12,13,14]. c) Iron bilayers with one of the monoclinic cells, and the corresponding supercell. Blue triangle illustrates geometrical frustration effects (see text). Red lines denote a minimal set of interactions [15 16] necessary for 3D charge order. and on a plausible microscopic model of a polar CO. This proposed "ferroelectricity from CO" is the main focus of the review, in particular recent findings by structure refinement of a non-polar CO [18] and the suggestion of the absence of polar order by dielectric spectroscopy [19,20]. Other aspects that have been of recent focus in these materials, such as geometrical frustration expressed in $\mathrm{CO}$ and magnetic order, strong spin-charge coupling, or potential electric-field-induced phase transitions, are treated as well. The main experimental findings reviewed are summarized in Sec. 7 which concludes with a brief outlook on future research directions both in this family of compounds and for "ferroelectricity from CO" in general.

2 The $\boldsymbol{R F e}_{2} \mathrm{O}_{4}$ family Rare earth ferrites $R \mathrm{Fe}_{2} \mathrm{O}_{4}$ with $R$ a $3+$ ion without partially occupied $d$ levels (Y, Ho, Er, Tm, Yb, Lu, or In), known since the 1970s [21], crystallize in a rhombohedral $(R \overline{3} m)$ structure [8,9,10, 11, 12, 13. 14] (see Fig. 19) featuring characteristic triangular bilayers of trigonal-bipyramidal-coordinated $\mathrm{Fe}$ as the electronically active subunit (c.f. Fig.11). Magnetism of the Fe spins attracted an initial wave of research into these materials, with unusual features such as anomalous thermomagnetization [22] or giant coercivity [23] discovered. In all investigated compounds, a very large Ising anisotropy with spins pointing perpendicular to the layers was observed [23, 24]. The average valence of the $\mathrm{Fe}$ ions is $2.5+$, and therefore $\mathrm{Fe}^{2+/ 3+}$ valence order or $\mathrm{CO}$ may also be expected, first investigated by Mössbauer spectroscopy [25]. Hence, there are two binary degrees of freedom at the $\mathrm{Fe}$ sites: Ising spin $\uparrow / \downarrow$ and valence $2+/ 3+$.

Geometrical Frustration The arrangement of $\mathrm{Fe}$ ions leads to "geometrical frustration" [26] hampering both spin order ( $\mathrm{SO}$ ) and $\mathrm{CO}$, illustrated in Fig. 1k) for

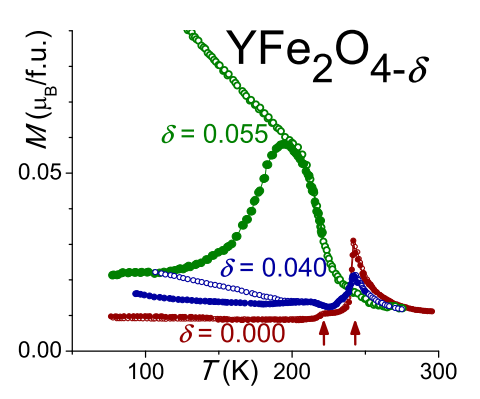

Figure 2 Magnetization $M(T)$ of polycrystalline $\mathrm{YFe}_{2} \mathrm{O}_{4-\delta}$ with different $\delta$, measured in $0.4 \mathrm{~T}$ upon heating, after the sample has been cooled in the same field (o) or in zero-field $(\bullet)$. Two transitions in the most stoichiometric sample are marked. Data from [27.

the Ising spins by a blue triangle: repulsive or antiferromagnetic (AF) nearest-neighbor (NN) interactions within the layers can be satisfied only for two of the spin pairs of the triangle. For an isolated layer, this would lead to a macroscopic degeneracy of ground states. The proximity of another triangular layer changes the topology of the problem, particularly since the inter-layer Fe-Fe NNdistance (marked $U_{2}$ in Fig. 11) is about $8 \%$ shorter than the intra-layer one (marked $U_{1}$ ). However, there is potential for frustration in the inter-layer interaction as well, because each $\mathrm{Fe}$ ion has three $\mathrm{NN}$ in the other layer (see also Fig. 8). Furthermore, the rhombohedral stacking leads to frustration of the interactions between different bilayers, hindering full 3D order. Even when macroscopic groundstate degeneracy is broken, geometrical frustration often [26] leads to a competition of different phases with nearly the same energy, facile creation of defects, and complex unusual ground states, such as the proposed [17] ferroelectric CO in these materials, discussed in Sec.4.

Strong impact of oxygen stoichiometry A soon observed experimental difficulty in these compounds was a strong variation of physical properties for different sam- 


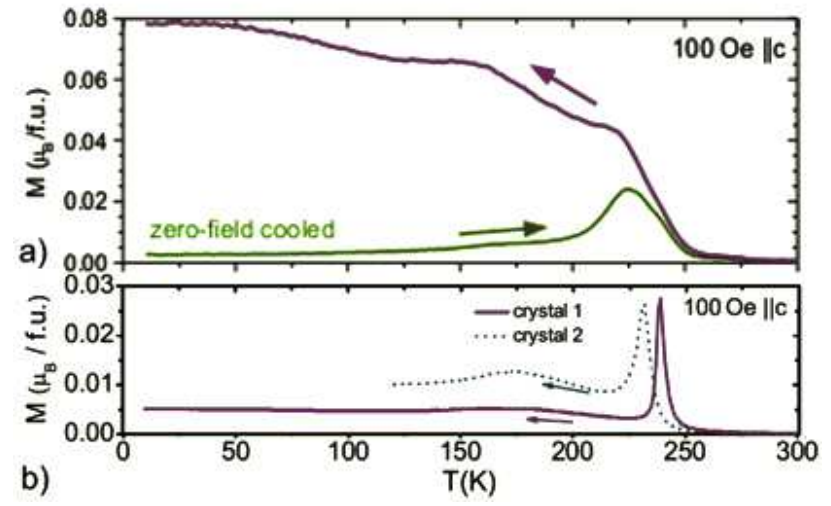

Figure 3 Magnetization $M(T)$ of three different $\mathrm{LuFe}_{2} \mathrm{O}_{4}$ crystals measured in 100 Oe $\| c$, upon cooling except where noted. Panel a) after [28], panel b) after [29].

ples. For $\mathrm{YFe}_{2} \mathrm{O}_{4-\delta}$ this was clearly linked to tiny variations in oxygen-stoichiometry [27], as shown in Fig. 2. magnetization $M(T)$ for the most off-stoichiometric sample shows a large difference between zero-field-cooled and field-cooled $M$ suggesting "glassy" magnetism without establishment of long-range $\mathrm{SO}$, whereas $M(T)$ for the stoichiometric sample indicates two distinct well-ordered antiferromagnetic phases. Single-crystal neutron diffraction confirmed a tendency towards 3D long-range order for samples closer to stoichiometry [30], but due to the absence of sufficiently stoichiometric crystals until recently [31], the magnetic structures remain unsolved. Such a sample-dependence was observed also for $\operatorname{Er}[32]$ and $\mathrm{Lu}$ (see Fig. 3), although the link to O-stoichiometry [33] is less clear, mostly due to the range of stoichiometries for which long-range order is observable shrinking with decreasing $R^{3+}$ size (c.f. [32] and Fig. 1b). In a wider range of stoichiometries, links to the remanent magnetization and resistivity are clear, however [34].

Ion-size effects and structural modifications Changing the $R^{3+}$ size leads to an expected change of the cell volume ( $\sim 5 \%$ between In and Y), but more relevant for $\mathrm{CO}$ and $\mathrm{SO}$ is a $15 \%$ change in the ratio between the layer-separation within a bilayer, $d_{b}$, and the intra-layer Fe-Fe distance $a_{\text {hex }}$ (Fig. 1 1 ), which can be expected to modify the relative importance of interactions $U_{1}$ and $U_{2}$. Partial substitution, e.g. by $\mathrm{Co}, \mathrm{Cu}$, or $\mathrm{Mn}$, on the $\mathrm{Fe}$ site generally leads to decreased conductivity, but also suppressed magnetic ordering (see e.g. [35,36]). However, effects due to substitution can be difficult to disentangle from effects due to a potentially changed O-stoichiometry. Finally, a larger structural modification can be done by intercalating $R \mathrm{Fe}_{2} \mathrm{O}_{4}$ with one or more blocks of $R \mathrm{FeO}_{3}$, each adding an additional $R$ - and a single-Fe layer between the bilayers [37]. There have been relatively few studies on these intercalated compounds (e.g. [38]). Finally, a high-pressure orthorhombic modification with a large supercell, possibly $\mathrm{CO}$, has recently been reported

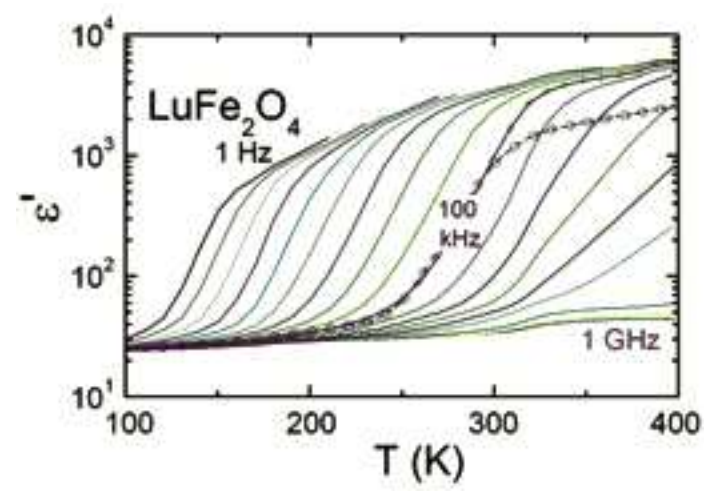

Figure 4 Real part of the dielectric permittivity $\varepsilon^{\prime}$ vs $T$ (lines) in $\mathrm{LuFe}_{2} \mathrm{O}_{4}$ for frequencies between $1 \mathrm{~Hz}$ and $1 \mathrm{GHz}$ equally spaced with two frequencies per decade. The data additionally marked with symbols $(0,+)$ are both taken at $100 \mathrm{kHz}$, but with electrodes from different material: Graphite (o) and silver ( + and all other data). Figure from [19], (c) 2012 American Physical Society.

[39], but the details of this structure have yet to be elucidated.

3 Anomalous dielectric dispersion and pyroelectric currents Many rare earth ferrites, e.g. $\mathrm{ErFe}_{2} \mathrm{O}_{4}$ [40], have been studied extensively by dielectric spectroscopy, with anomalously large real parts of the dielectric constant $\varepsilon^{\prime}$ in the low-frequency limit generally observed. A typical (recent) example of such a measurement is shown in Fig. 4 From the frequency-dependence of the temperature of maximum rise of $\varepsilon^{\prime}(T)$ a connection of the dispersion to electrons hopping between $\mathrm{Fe}^{2+}$ and $\mathrm{Fe}^{3+}$ has been concluded and an origin of the large dielectric constants in motion of ferroelectric domain boundaries suggested [17,40]. Within this interpretation, giant (>20\%) roomtemperature magneto-dielectric response [41] would then suggest multiferroicity with large magnetoelectric coupling, potentially useful for applications. Research on possible ferroelectricity in rare earth ferrites focused on $\mathrm{LuFe}_{2} \mathrm{O}_{4}$, because in this compound superstructure reflections were found, which for example by resonant $\mathrm{x}$-ray diffraction at the $\mathrm{Fe} K$-edge could be clearly associated with $\mathrm{Fe}^{2+/ 3+}$ charge order [17,42], allowing the discussion of potentially polar CO phases (see Sec. 4).

Macroscopic proof of ferroelectricity requires demonstration of a remanent electric polarization, which should be switchable by application of an electric field. A remanent polarization larger than in the traditional ferroelectric $\mathrm{BaTiO}_{3}$ was suggested by pyroelectric current measurements [17], shown in Fig. 5]). These measurements were performed by first cooling the single-crystal sample in an electric field of $\pm 10 \mathrm{kV} / \mathrm{cm}$, then switching off the electric field, and finally measuring, upon warming in $E=0$, the pyroelectric current associated with the change of the 


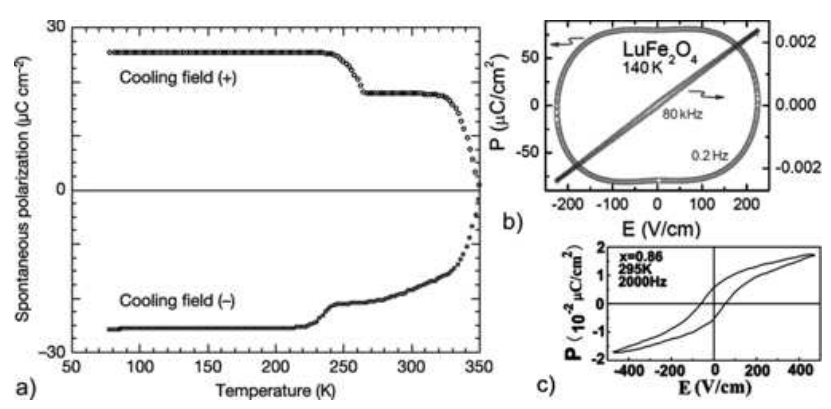

Figure 5 a) Electric polarization $P$ vs temperature $T$ of $\mathrm{LuFe}_{2} \mathrm{O}_{4}$ as deduced from pyroelectric current measurements conducted on warming after the sample had been cooled in electric fields $E \| c$ of $\pm 10 \mathrm{kV} / \mathrm{cm}$. Panel from [17], (c) 2005 Nature Publishing Group. b) $P$ vs $E$ hysteresis loops of $\mathrm{LuFe}_{2} \mathrm{O}_{4}$ at $140 \mathrm{~K}$ for two different frequencies, from [20], (C) EDP Sciences, Società Italiana di Fisica, Springer-Verlag 2012. With kind permission of The European Physical Journal (EPJ). c) $P(E)$ of $\mathrm{Lu}_{2} \mathrm{Fe}_{2.14} \mathrm{Mn}_{0.86} \mathrm{O}_{7}$ at $2 \mathrm{kHz}$, from [38], (C) 2009 American Institute of Physics.

polarization. The dependence of the pyroelectric current and thus the polarization on the direction of the cooling field is consistent with a macroscopic polarization, and the decay to zero around the charge ordering temperature of $T_{C O} \sim 320-330 \mathrm{~K}$ [43,44] supports a connection of $P$ with the charge ordering, although the lack of a saturation region with no pyrocurrent flowing anymore above $T_{C O}$ renders this last conclusion tentative. It was noted, however, that similar behavior can also be observed in (nonferroelectric) leaky dielectrics, where it originates from localization of free carriers at interfaces [45].

Subsequent measurements by various groups partly reproduced the pyrocurrent results of Fig. 5 a), as noted e.g. in [42], or tried to establish $P(E)$ hysteresis loops at low temperatures to more directly show ferroelectricity. Out of many attempts at the latter, only few were published [20, 46 47, some showing hysteresis, but not the saturation necessary [48] to establish polarization switching. Fig. 5b) shows $P(E)$ measured at $140 \mathrm{~K}$ and two frequencies [20]: the low-frequency loop shows substantial hysteresis, but no indications of saturation, whereas the loop measured with a frequency of $80 \mathrm{kHz}$ exhibits practically linear behavior without appreciable hysteresis.

Because the polarization is linked with the dielectric constant, $\mathbf{P}=(\varepsilon-1) \varepsilon_{0} \mathbf{E}$, detailed modeling of the dielectric dispersion can show the origin of observed polarization behaviors. This was done for the results obtained on $\mathrm{LuFe}_{2} \mathrm{O}_{4}$ single crystals over 9 decades of frequencies [19] and shown in Fig. 4. The qualitative behavior with large $\varepsilon^{\prime}$ at low frequency and high $T$ is the same as in previous observations [17], but a strong impact of the electrode material as shown for the example of $100 \mathrm{kHz}$ (different symbols) already suggests significant contact effects, e.g. due to Schottky-type depletion layers at the contact interfaces, which act as an additional capacitance [19]. The whole data-set of $\varepsilon^{\prime}(f, T)$ and $\varepsilon^{\prime \prime}(f, T)$ could be well described with an equivalent-circuit model featuring contactcapacitance and -conductance, and sample hopping- and dc-conductivity, in addition to the intrinsic dielectric constant $\varepsilon_{i}$ of the material, all parameters independent of frequency. The fitted $\varepsilon_{i}(T)$ is only around 30 and does not show any indications of ferroelectric or antiferroelectric transitions. Two recent studies on polycrystalline $\mathrm{LuFe}_{2} \mathrm{O}_{4}$ reached the same conclusion [20,49]. For low frequencies or high $T$, the circuit reduces to a leaky contact capacitance in series with the sample resistivity, resulting in large $\varepsilon^{\prime}$. Because the step in $\varepsilon^{\prime}$ mainly depends on contact capacitance and sample resistance [19] a magneto-resistance will lead to an apparent magnetodielectric response, according to the analysis in [49] the latter can be fully accounted for by the former. A very recent study [50] of dielectric spectroscopy using various contact materials suggests a small ( 1\%) sample magnetodielectric effect at least at $220 \mathrm{~K}$ (at the AFM-fM transition, c.f. Fig. 11), but the simultaneously observed 18 times larger change in resistivity may influence the fitted sample capacity.

$P(E)$ loops can only show the intrinsic behavior if measured at frequencies and temperatures where $\varepsilon^{\prime}(f, T)$ is close to $\varepsilon_{i}(T)$. For the measurements shown in Fig. 5b), this is the case for $P(E)$ measured at $80 \mathrm{kHz}$, but clearly not for $P(E)$ measured at $0.2 \mathrm{~Hz}$. There is one $P(E)$ loop in the literature [38] that does seem to indicate ferroelectricity given the expected tendency to saturate in high $E$ (Fig. 56), measured on intercalated $\mathrm{Lu}_{2} \mathrm{Fe}_{3} \mathrm{O}_{7}$, in which conductivity has been suppressed by $\sim 29 \% \mathrm{Mn}$ for Fe substitution. The occurrence of at least piezoelectricity in this compound is supported by piezoresponse force microscopy [51]. Although the switchable polarization is very weak (compare panels c and a), smaller even than in "spin-spiral multiferroics" like $\mathrm{TbMnO}_{3}$, this suggests that intercalated compounds deserve more attention than they currently get.

4 Charge Order It was the combination of macroscopic indications of ferroelectricity, discussed in Sec. 3 , with a likely microscopic model of charge order (CO) that is electrically polar, which made a convincing case for $\mathrm{LuFe}_{2} \mathrm{O}_{4}$ exhibiting ferroelectricity from $\mathrm{CO}$ [17]. This $\mathrm{CO}$-model was deduced by scattering methods, in which signatures of the order in reciprocal space are investigated.

For many $R \mathrm{Fe}_{2} \mathrm{O}_{4}$, at least diffuse scattering of x-rays at the $\left(\frac{1}{3} \frac{1}{3} \ell\right)$ line in reciprocal space (in hexagonal-cell notation) suggested a tendency towards a $\mathrm{CO}$ that could be described, for example, with a so-called $\sqrt{3} \times \sqrt{3}$ cell. Alternatively, a propagation vector with $\left(\frac{1}{3} \frac{1}{3}\right)$ in-plane component can be conveniently described by expressing the crystal structure without $\mathrm{CO}$ in a $C$-centered monoclinic cell, which is then enlarged three times along its $b$-axis. Because such a $\mathrm{CO}$ breaks the 3 -fold rotation symmetry of the crystal structure, domains of $\mathrm{CO}$ with symmetry- 


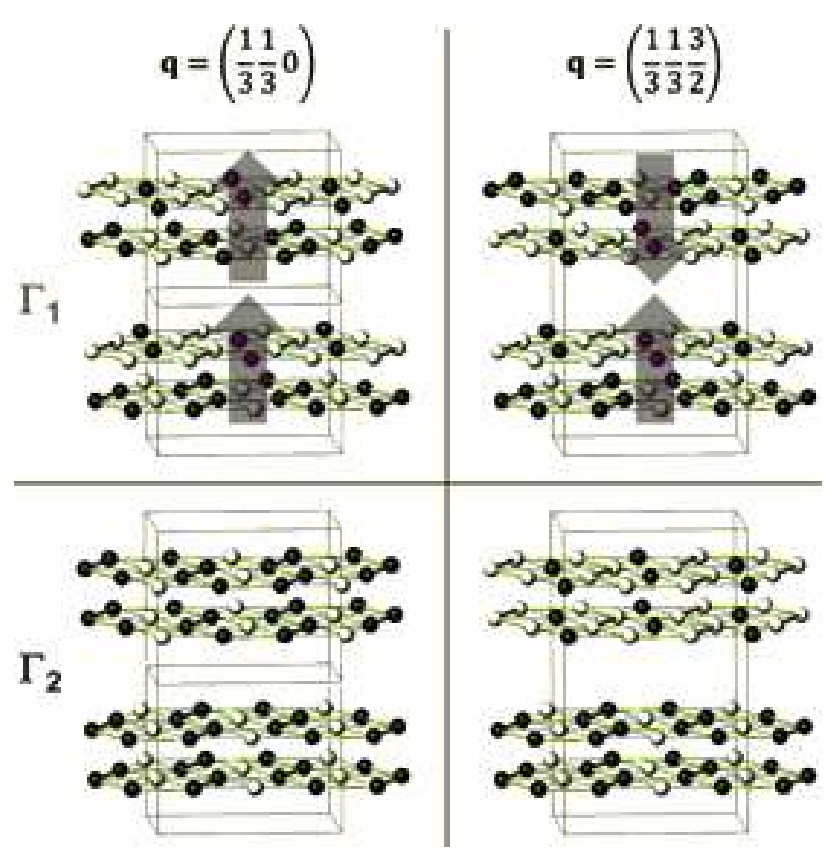

Figure 6 Sketch of the 4 symmetry-allowed $\mathrm{Fe}^{2+}$ (black) $/ \mathrm{Fe}^{3+}$ (white) charge configurations following $\left(\frac{1}{3} \frac{1}{3} 0\right)$ or $\left(\frac{1}{3} \frac{1}{3} \frac{3}{2}\right)$ propagation (with additional contribution by (000) or $\left(00 \frac{3}{2}\right)$, respectively). Arrows indicate bilayer polarization.

equivalent $\left(\frac{2}{3} \frac{1}{3}\right)$ and $\left(\frac{1}{3} \frac{2}{3}\right)$ propagations and $120^{\circ}$ rotated monoclinic cells, as sketched in Fig. 1] also exist [44].

The simplest arrangement of $\mathrm{Fe}^{2+}$ and $\mathrm{Fe}^{3+}$ ions in a bilayer leading to such propagations and maintaining charge neutrality is the one proposed in [17] and shown in Fig. 6 top-left (two cells of the $\mathrm{CO}$ are shown). Here, for each bilayer, the upper layer has a surplus of $\mathrm{Fe}^{3+}$ ions and the lower layer a surplus of $\mathrm{Fe}^{2+}$ ions, which implies an electric dipole moment between the layers (indicated by arrows), i.e. this $\mathrm{CO}$ is indeed ferroelectric, rendering the bilayers polar.

4.1 Long-range $\mathbf{C O}$ from diffraction In the case of $\mathrm{LuFe}_{2} \mathrm{O}_{4}$, actual superstructure reflections were observed, below $T_{\mathrm{CO}} \sim 320 \mathrm{~K}$, by several groups using various diffraction techniques (Fig. 7). Most studies resolving $3 \mathrm{D}$ peaks found the principal superstructure reflections to be near positions ( $\frac{1}{3}, \frac{1}{3}$,halfinteger) and symmetryequivalent [17, 18, 15, 42, 43, 44, 52, 53, 54, 55, 56, 57, 58, 59], with in-plane/out-of-plane correlation lengths given as e.g. $30 / 6 \mathrm{~nm}$ [53] or 80/7 nm [55]. Resonant X-ray diffraction at the $\mathrm{Fe} \mathrm{K}$-edge of these reflections support their association with $\mathrm{Fe}^{2+/ 3+} \mathrm{CO}[17,42,54,58]$. Sharp reflections at other positions, including near $\left(\frac{1}{3}, \frac{1}{3}\right.$,integer $)$ [60,61,62] are sometimes observed in electron diffraction studies, generally on samples with higher oxygen content [59,62]. The additional reflections in [59] were attributed to ordering of excess oxygen ions.
High-resolution x-ray diffraction [15,43,44] found the exact positions of the $\left(\frac{1}{3}, \frac{1}{3}\right.$, halfinteger) reflections to be well described by $\mathbf{s}+\mathbf{p}_{\mathbf{A}}, \mathbf{s}+\mathbf{p}_{\mathbf{B}}$, and $\mathbf{s}+\mathbf{p}_{\mathbf{C}}$, where $\mathbf{s}$ is a structural reflection following the rhombohedral centering condition $-h+k+\ell=3 n$ with $n$ integer. The three symmetry-equivalent propagation vectors, corresponding to CO domains, are $\mathbf{p}_{\mathbf{A}}=\left(\frac{1}{3}+\delta, \frac{1}{3}+\delta, \frac{3}{2}\right)$, $\mathbf{p}_{\mathbf{B}}=\left(\frac{\overline{2}}{3}-2 \delta, \frac{1}{3}+\delta, \frac{3}{2}\right)$, and $\mathbf{p}_{\mathbf{C}}=\left(\frac{1}{3}+\delta, \frac{\overline{2}}{3}-2 \delta, \frac{3}{2}\right)$ and $\delta \sim 0.003 T$-dependent [44]. A secondary set of reflections is described by $\mathbf{p}_{\mathbf{A}}^{\prime}=\left(\tau, \tau, \frac{3}{2}\right), \mathbf{p}_{\mathbf{B}}^{\prime}=\left(\overline{2 \tau}, \tau, \frac{3}{2}\right)$, and $\mathbf{p}_{\mathbf{C}}^{\prime}=\left(\tau, \overline{2 \tau}, \frac{3}{2}\right)$, and $\tau \sim 9 \delta$ following the $T$-dependence of $\delta$. Although this seems to suggest an incommensurate modulation of the $\mathrm{Fe}$ valence state, there is no evidence of a "true" incommensuration, which would imply a wide distribution of $\mathrm{Fe}$ valence states from $2+$ to $3+$, in conflict with spectroscopic measurements [25, 63, 64]. Much more likely is therefore a locally commensurate state, interspersed with discommensurations or anti-phaseboundaries (Fig. 8) of an average separation, estimated from $\tau \sim 0.028$ [44], of about $12 \mathrm{~nm}$. It has been demonstrated on other charge-ordering oxides that a proliferation of such anti-phase-boundaries, which can cost very little energy due to geometrical frustration, can lead to sharp superstructure reflections at incommensurate positions [65].

Consequently, the possible $\mathrm{CO}$ corresponding to $\left(\frac{1}{3} \frac{1}{3} \frac{3}{2}\right)$ and for completeness also $\left(\frac{1}{3} \frac{1}{3} 0\right)$ have to be examined through symmetry-analysis [66]. For each of these propagation vectors, there are two irreducible representations, corresponding to the two $\mathrm{Fe}$ ions of the primitive unit cell (labeled 1 and 2 in Fig. 1a) having the same $\left(\Gamma_{2}\right)$ or different $\left(\Gamma_{1}\right)$ valence. Each corresponding $\mathrm{CO}$ contains more than than two valence states, in contradiction to the results of Mössbauer spectroscopy, which imply a bimodal valence distribution [63]. Such a bimodal distribution is obtained by combining, in a unique way [44], the CO from $\left(\frac{1}{3} \frac{1}{3} \frac{3}{2}\right)$ [resp. $\left.\left(\frac{1}{3} \frac{1}{3} 0\right)\right]$ with the one from $\left(00 \frac{3}{2}\right)$ [resp. (000)].

The four resulting $\mathrm{CO}$ are shown in Fig. 6, The $\mathrm{CO}$ with $\Gamma_{2}$ (lower row) have bilayers with a net charge, indeed for $\left(\frac{1}{3} \frac{1}{3} 0\right) /(000)$ the whole structure would be charged. $\left(\frac{1}{3} \frac{1}{3} 0\right) /(000)$ with $\Gamma_{1}$ gives the ferroelectric CO with polar bilayers proposed in [17]. However, since the observed (also in [17]) superstructure reflections follow $\left(\frac{1}{3} \frac{1}{3} \frac{3}{2}\right)$ propagation, one of the two CO shown on the right of Fig. 6 should be realized, either (top) the antiferroelectric $\mathrm{CO}$ with polar bilayers but zero net polarization, as proposed in [44], or (bottom) the CO with stacking of oppositely charged bilayers. The latter was initially dismissed because it involves a transfer of charge between neighboring bilayers separated by $\sim 6 \AA$, but an experimental determination of which $\mathrm{CO}$ is realized requires a full structural refinement.

This was recently achieved [18], the key being the screening of a large quantity of small crystals of the highest quality, as judged by $M(T)$ (Fig. 3), for one with almost only one of the three possible CO-domains populated. The structure obtained at $210 \mathrm{~K}$, readily refined in the space 

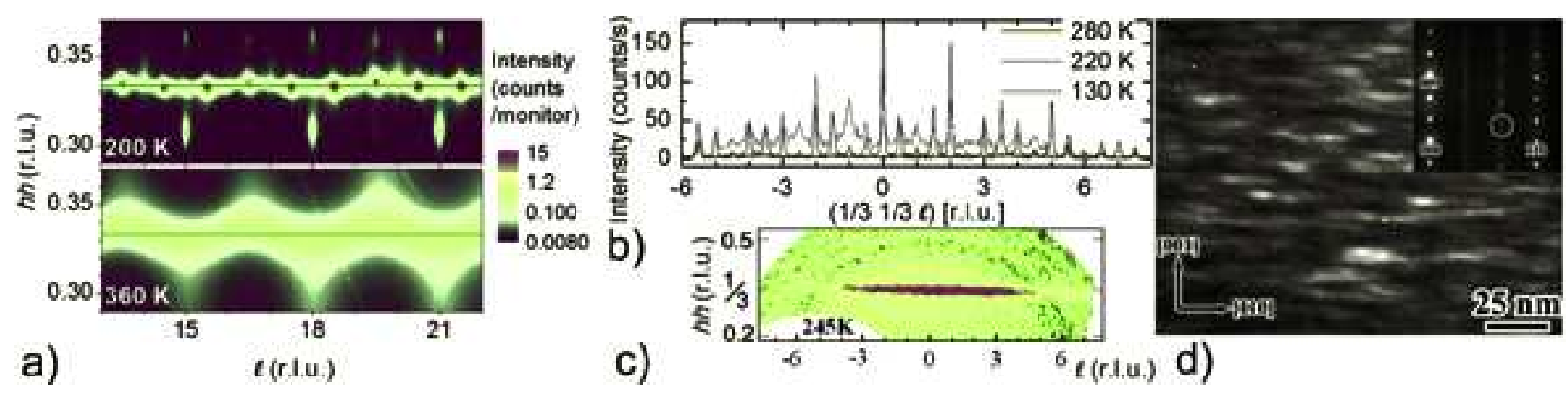

Figure 7 Charge order $\mathrm{CO}$ superstructure in $\mathrm{LuFe}_{2} \mathrm{O}_{4}$ by different techniques. a) X-ray diffraction scattered intensity at ( hh $\ell$ ) at 200 and 360 K. From [44], (c) 2008 American Physical Society. b) Neutron diffraction scans along $\left(\frac{1}{3} \frac{1}{3} \ell\right)$ at three temperatures. From [52], (c) 2008 American Physical Society. c) Neutron diffraction intensity in spin-flip channel at ( $h h \ell$ ) at 245 K. From [29], (C) 2012 American Physical Society. d) Electron-diffraction dark-field image taken at $92 \mathrm{~K}$ with a $\left(\frac{1}{3} \frac{1}{3} \frac{5}{2}\right)$ superstructure spot, circled in the inset. The CO domains extend $\sim 30 \mathrm{~nm}$ in the $a b$-plane and $\sim 6 \mathrm{~nm}$ along $c$. Inset: electron diffraction pattern with (11̄0) incidence. From [53], (c) 2009 American Physical Society.

group $C 2 / m$ with a residual of $5.96 \%$, contains four ironsites with valences, determined with the bond-valence-sum (BVS) method [67], 1.9, 2.1, 2.8, and 2.9 [18]. Given the uncertainties inherent to the empirical BVS-method, these values can be considered to be remarkably close to the ideal values of 2 and 3 (for a comparison of other charge ordering ferrites see Fig. 5 in [68]). The thus identified CO is the one with charged bilayers, shown in Fig. 6 bottom right. Attempts to force-refine the other $\mathrm{CO}$ or to refine in a lower-symmetry space group confirmed this structure solution, and independent confirmation was also obtained indirectly via spin-charge coupling (c.f. Sec. 5.3). The groundstate $\mathrm{CO}$ of $\mathrm{LuFe}_{2} \mathrm{O}_{4}$ is thus not ferroelectric and does not contain the initially proposed polar bilayers.

4.2 Short-range correlations and charge dynamics Above $T_{\mathrm{CO}}$, the sharp $\mathrm{CO}$ superstructure reflections in $\mathrm{LuFe}_{2} \mathrm{O}_{4}$ are replaced by diffuse scattering along $\left(\frac{1}{3} \frac{1}{3} \ell\right)$, with a characteristic zig-zag pattern, shown in Fig. 7 7 ) lower graph, discernable up to about $550 \mathrm{~K}[43,61]$. A detailed analysis of the scattered intensity [44] suggests that it can be described as very broad and overlapping peaks at positions of structural reflections $\pm\left(\frac{1}{3}-\delta^{\prime}, \frac{1}{3}-\right.$ $\left.\delta^{\prime}, 0\right)$ and symmetry-equivalent. Corresponding to the large

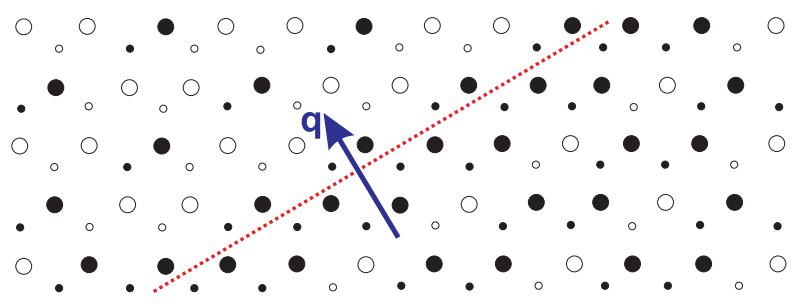

Figure 8 Example of a possible anti-phase-boundary (dashed) perpendicular to the in-plane propagation of $\mathrm{CO}$, which switches the majority valence. Top-view of bilayer $\left(\bullet=\mathrm{Fe}^{2+}, \mathrm{o}=\mathrm{Fe}^{3+}\right), \mathrm{Fe}$ ions in the lower layer are drawn smaller. peak-width along $\ell$, at $360 \mathrm{~K}$ a correlation extending only to $2-3$ bilayers was concluded, whereas the remaining relative sharpness along ( $h h 0)$ suggests that in each individual bilayer, medium-range $\mathrm{CO}$ is maintained much above $T_{\mathrm{CO}}$. Applying symmetry-analysis on the positions of the diffuse peaks suggests a marginal tendency towards ferrostacking of neighboring bilayers, one of the $\mathrm{CO}$ shown in Fig. 6 left. In [44], representation $\Gamma_{1}$ and thus a tendency towards ferroelectric CO, was assumed. However, as it is now clarified that in the long-range ordered state, representation $\Gamma_{2}$ with charged rather than polar bilayers is realized, it seems more likely that the short-range $\mathrm{CO}$ is also following $\Gamma_{1}$, without polar bilayers ever being present.

This seems at first impossible, as this corresponds to an overall net charge of the structure. However, given the small correlation-volume deduced from the broadness of the diffuse peaks, a charge-transfer over a few bilayers (via a presently unknown pathway) is sufficient, and feasible considering that this is not a static, but a dynamic short-range $\mathrm{CO}$, i.e. short-range-correlated valence fluctuations. Mössbauer spectroscopy can directly assess the dynamics of $\mathrm{CO}$ and charge fluctuations: spectra of several $R \mathrm{Fe}_{2} \mathrm{O}_{4}$ were analyzed with a model featuring isomer shifts, quadrupole splittings, and linewidths for both valence states and additionally a Fe ${ }^{2+} \rightarrow \mathrm{Fe}^{3+}$ hopping frequency, showing still discernable natural hopping even somewhat below $T_{\mathrm{CO}}[25]$.

Fig. 97) shows the hopping frequency $\nu_{\text {hopping }}$ determined in a study on powdered crystals of optimal quality. The hopping above $T_{\mathrm{CO}}$ can be described by an Arrhenius law with an activation energy of $\sim 0.16 \mathrm{eV} . \nu_{\text {hopping }}$ decreases continuously upon cooling through $T_{\mathrm{CO}}$, and below can again be described by an Arrhenius law, but with a higher activation energy $\sim 0.36 \mathrm{eV}$. The Arrhenius law with a change of slope at $T_{\mathrm{CO}}$ is consistent with the $T$-dependence of the dc conductivity extracted by dielectric spectroscopy on similar samples [19]. The substantial 

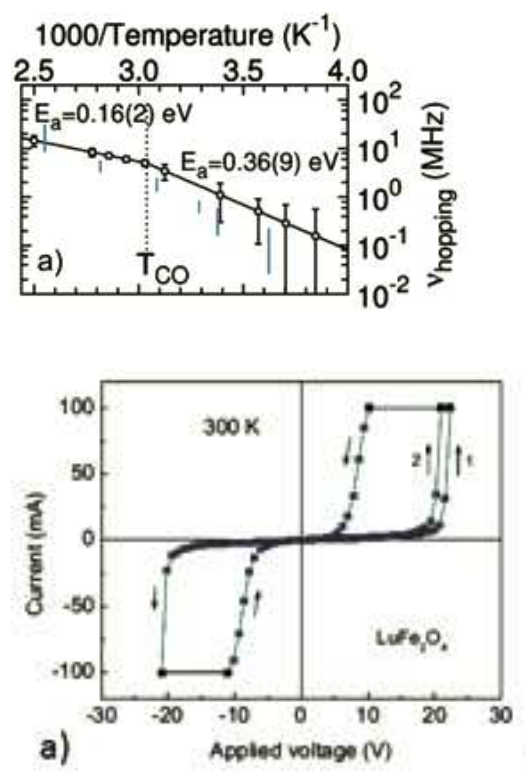
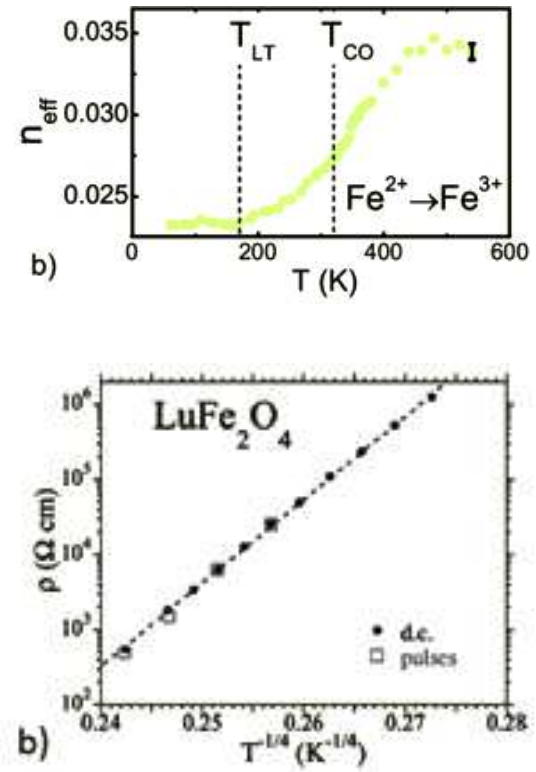

Figure 9 Charge dynamics in $\mathrm{LuFe}_{2} \mathrm{O}_{4}$. From [63], (C) 2008 American Physical Society. a) Arrhenius-plot of the $\mathrm{Fe}^{2+} \rightarrow \mathrm{Fe}^{3+}$ hopping frequency determined from Mössbauer spectra (o, bars are from [25]). b) Temperaturedependence of the strength $n_{\text {eff }}$ of an optical excitation assigned to $\mathrm{Fe}^{2+} \rightarrow \mathrm{Fe}^{3+}$ charge transfer.

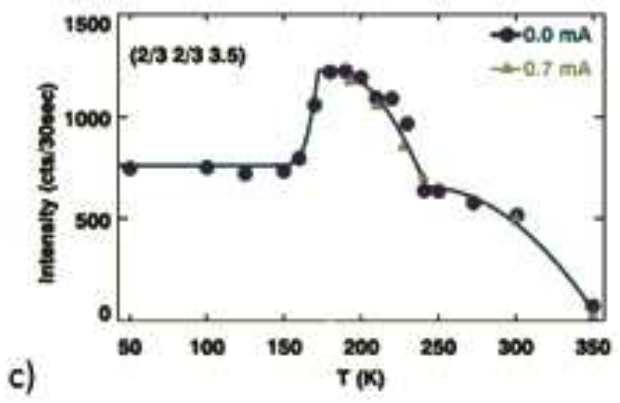

Figure 10 Electric-field effects in $\mathrm{LuFe}_{2} \mathrm{O}_{4}$. a) Current-voltage hysteresis loop at $300 \mathrm{~K}$, with the current limited to $100 \mathrm{~mA}$. From [69], (C) 2008 American Institute of Physics. b) Resistivity $\rho$ vs $T^{-1 / 4}$. full symbols: low $E$-field d.c. measurements; open symbols: high-field pulsed measurements. From [70], (C) 2011 American Institute of Physics. c) Neutron diffraction intensity of $\left(\frac{2}{3} \frac{2}{3} \frac{7}{2}\right)$ as a function of calibrated temperature, obtained by cooling with $0.7 \mathrm{~mA}(\triangle)$ or no current (•). From [56], (c) 2010 American Physical Society.

electron hopping present even below $T_{\mathrm{CO}}$, where diffraction indicates long-range $\mathrm{CO}$, likely involves fluctuations of anti-phase boundaries, a scenario shown to be at work in another $\mathrm{CO}$ ferrite, and connected to $T$-dependent apparent incommensuration [65]. In $\mathrm{LuFe}_{2} \mathrm{O}_{4}$ the incommensuration becomes constant only below $\sim 170 \mathrm{~K}$, suggesting that the charge dynamics become completely frozen only at temperatures substantially below $T_{\mathrm{CO}}$.

The capacity of $\mathrm{Fe}^{2+} \rightarrow \mathrm{Fe}^{3+}$ charge transfer can be probed not only through natural electron hopping, but also by driving the process at optical frequencies. Fig. $9 \mathrm{~b})$ shows the $T$-dependence of the strength of an optical excitation assigned to $\mathrm{Fe}^{2+} \rightarrow \mathrm{Fe}^{3+}$ charge transfer [63]. Upon cooling, it starts to decrease around $500 \mathrm{~K}$, reasonably close to the reported [43,61] onset of diffuse scattering, and it bottoms out only at $T_{\mathrm{LT}} \sim 170 \mathrm{~K}$, consistent with the freezing of charge dynamics at this temperature. The nature of the phase below $T_{\mathrm{LT}}$ will be further discussed in Sec.6.2.

\subsection{Electric-field and current effects on $\mathrm{CO}$}

Given that the pyroelectric current measurements shown in Fig. 5a) had been prepared by first cooling the sample in an electric field, and that diffraction analyses failed to deduce a ferroelectric $\mathrm{CO}$, it had been proposed [44] that such a ferroelectric $\mathrm{CO}$ might be stabilized by electric fields $E$ or by cooling in $E$. However, at low $T$ electric fields up to $20 \mathrm{kV} / \mathrm{cm}$ were shown to have no effect on the $\mathrm{CO}$ [56]. At higher $T$, intriguing electric-field effects have been observed macroscopically in the form of non- linear current-voltage characteristics (Fig. 10, [69,71]: Rather than eventually reaching a state with lower resistance as might be expected for a ferroelectric phase, a current-breakthrough is observed. The breakthrough depends on the environment so that a possible application in gas-sensing has been proposed [72]. Effects were also reported on dielectric constants [73] and magnetism [57, 74,75]. With in-situ electron diffraction experiments, a disappearance of the $\mathrm{CO}$ superstructure reflections at this threshold was observed [76,77], suggesting an electricfield or current-induced melting of the $\mathrm{CO}$. In contrast, an ex-situ in-field cooling experiment suggests improved $\mathrm{CO}$ correlations, without affecting the intensity-distribution between different reflections [78].

However, current-voltage measurements using single short ( $\sim 1 \mathrm{~ms}$ ) current pulses [70] found Ohmic behavior in polycrystalline $\mathrm{LuFe}_{2} \mathrm{O}_{4}$ (see Fig. 10p) up to $E$ much higher than fields where the breakdown in dc measurements occurs, with the time-dependence on longer pulses suggesting self-heating effects [70]. Similar pulsed measurements on $\mathrm{YbFe}_{2} \mathrm{O}_{4}$ [79] suggest non-linearity in the in-plane conductivity, attributed to sliding charge density and possibly a break-down of $\mathrm{CO}$, whereas large selfheating effects for longer pulses are also found. An in-situ neutron diffraction experiment [56] demonstrates the absence of any current-dependence on either charge or magnetic order when the sample-temperature is properly recalibrated (Fig. 10k). A CO unaffected by static (melting of the $\mathrm{CO}$ by $1.55 \mathrm{eV}$ photons was demonstrated by a re- 
cent pump-probe experiment [80]) electric fields or currents was also found by in-situ infrared spectroscopy [81], by in-situ $\mathrm{x}$-ray diffraction with the real sample temperature established from the thermal expansion of the silver electrodes [82], and by resonant $\mathrm{x}$-ray diffraction after infield cooling [58]. Although a recent infrared-spectroscopy study [83] suggests very subtle field-effects not attributable to self-heating, the conclusion that a different, possibly ferroelectric, $\mathrm{CO}$ cannot be stabilized by electric fields is unavoidable.

4.4 Theoretical considerations The experimentally deduced $\mathrm{CO}$ with charged bilayers is surprising, because $\mathrm{CO}$ is usually assumed to be driven by the repulsion of electrons between different sites ("Wigner crystallization"), which should result in minimizing the occurrence of neighbors of the same valence. It is clear that packing electrons close together in a charged bilayer should lead to a significant energy penalty. Although the full description of the $3 \mathrm{D}$ CO requires at least the four interactions $U_{1}-U_{4}$ indicated in Fig. 1, [15, 16], the clear indications by the diffuse scattering above $T_{\mathrm{CO}}$ (Sec. 4.2) that correlations within the bilayers are much stronger than those between different bilayers make a first analysis based on only $U_{1}$ and $U_{2}$ (intrabilayer interactions) useful. Concerning the intralayer interaction $U_{1}$ all the $\mathrm{CO}$ of Fig. 6, as well as many others, such as a stripe-like $\mathrm{CO}$, are exactly degenerate due to geometrical frustration (c.f. Sec. 2). However, concerning the three nearest neighbors $(\mathrm{NN})$, which are in the other layer $\left(U_{2}\right)$, the charged bilayer $\mathrm{CO}$ (Fig. 6 bottom) have on average $\frac{5}{3}$ same-valence $\mathrm{NN}$, whereas the polar CO (Fig. 6 top) have only $\frac{4}{3}$ same-valence NN, and is therefore more stable for repulsive $U_{2}$. However, a stripe-like $\mathrm{CO}$ can have 1 same-valence $\mathrm{NN}$, which should make it even more favorable, as found also by Monte-Carlo simulations taking into account the long-range nature of the Coulomb interaction [84]. This is an indication that Coulomb repulsion is not the sole relevant driving force for $\mathrm{CO}$ in $R \mathrm{Fe}_{2} \mathrm{O}_{4}$. It is not really surprising: a proof of purely electrostatically driven $\mathrm{CO}$ has been elusive in bulk materials and strong influence of lattice distortions on $\mathrm{CO}$ are well known, e.g. in manganites [85]. In addition to lattice effects, indicated to be relevant in $\mathrm{LuFe}_{2} \mathrm{O}_{4}$ by infrared [7, 86] and Raman [87] spectroscopy, CO driven by magnetic exchange has also been demonstrated [88].

Density-functional-theory (DFT) calculations performed for $\mathrm{LuFe}_{2} \mathrm{O}_{4}$ [84] suggest a lattice-contribution of about $40 \%$ to the total energy gain by $\mathrm{CO}$ for the ferroelectric ( $402 \mathrm{meV} /$ f.u.) and stripe $(384 \mathrm{meV} /$ f.u. $) \mathrm{CO}$ investigated, estimated by separately stabilizing the $\mathrm{CO}$ with fixed atomic positions and with relaxing the structure. Charged bilayer or antiferroelectric CO could not be obtained, because a cell with 3 bilayers was used. A ferrielectric $\mathrm{CO}$ with a $\uparrow \downarrow \uparrow$ stacking of bilayer polarizations (and a net polarization close to the one indicated in Fig. 5a, lending support to this model) and later using a larger cell an antiferroelectric CO [44] was found to be yet more stable, by a total of $15 \mathrm{meV} / \mathrm{f} . \mathrm{u}$. . These small energy differences between antiferroelectric, ferroelectric, and stripe $\mathrm{CO}$ are an indication of the relevance of geometrical frustration, as expected (Sec. 2). Spin-orbit coupling and magnetism was considered in a later study [89] (see Sec. 5.3). In this study, a fixed ferroelectric $\mathrm{CO}$ was used, but the reported energy gain of $78 \mathrm{meV} / \mathrm{f}$.u. is considerably larger than the above energy-differences between different $\mathrm{CO}$, indicating that magnetism may be relevant in stabilizing the $\mathrm{CO}$. The charged bilayer $\mathrm{CO}$ has yet to be tested by DFT.

The $\mathrm{CO}$ in $R \mathrm{Fe}_{2} \mathrm{O}_{4}$ has also been studied by lattice gas models. Modeling a single bilayer [90, 91, 92, 93, 94 already yields several competing $\mathrm{CO}$ phases, among them the ferroelectric $\mathrm{CO}$ (Fig. 6 top left) and non-polar $\mathrm{CO}$ phases with in-plane propagations such as $\left(\frac{1}{2} 0\right),\left(\frac{1}{4} \frac{1}{4}\right),\left(\frac{1}{6} \frac{1}{6}\right)$ and $\left(\frac{5}{12} \frac{5}{12}\right)$, again pointing out the importance of geometrical frustration. Because in $90,91,92,93,94$, charge neutrality of this bilayer was implied, the charged bilayer $\mathrm{CO}$ was not considered. A lattice gas model defined on the whole 3D lattice of $\mathrm{Fe}$ has been considered in [15, 16], though without considering in detail the charged bilayer $\mathrm{CO}$ found experimentally. Starting point is the Hamiltonian

$$
\mathcal{H}=\sum U_{i j}^{\nu \nu^{\prime}} \cdot \sigma_{i}^{\nu} \sigma_{j}^{\nu^{\prime}}
$$

where $i / j$ enumerate the primitive unit cells, $\nu / \nu^{\prime}=1,2$ denote the $\mathrm{Fe}$ ion in the primitive cell, $\sigma$ assumes the value $1(-1)$ for the valence state $2+(3+)$ of the Fe ion, and $U$ denotes screened Coulomb-interactions between different $\mathrm{Fe}$ ions. In reciprocal space, the eigenvalues of the Fourier-transformed Hamiltonian (1) give the energies for charge patterns defined by a corresponding eigenvector and a propagation vector, and the charge pattern with the lowest energy corresponds to the realized CO. Four interactions ( $U_{1}-U_{4}$ in Fig. 1p) are considered. It is clear that at least interactions $U_{1}-U_{3}$ are needed to yield a $3 \mathrm{D} \mathrm{CO}$ : They lead to instabilities at $\left(\frac{1}{3} \frac{1}{3} \ell\right)$, but these are degenerate along $\ell$, yielding only 2D CO [15]. This is due to the rhombohedral stacking of the bilayers [16], a geometrical frustration effect.

With a non-zero $U_{4}$, depending on the signs of $U_{4}$ and $U_{2} \cdot U_{3}$ possible ordering wavevectors $\left(\frac{1}{3} \pm \delta, \frac{1}{3} \pm \delta, \frac{3}{2}\right)$ or $\left(\frac{1}{3} \pm \delta, \frac{1}{3} \pm \delta, 0\right)$ are obtained. $U_{4}<0$ (attractive, i.e. favoring same valence) must be assumed to yield the observed propagation vector, which was proposed to be due to overscreening [15]. The observed (c.f. Sec. 4.2) discrepancy between the dominant fluctuations above $T_{\mathrm{CO}}$ with peaks near $\left(\frac{1}{3}, \frac{1}{3}\right.$,integer) and the long-range $\mathrm{CO}$ below $T_{\mathrm{CO}}$ with reflections near $\left(\frac{1}{3}, \frac{1}{3}\right.$,half-integer) is difficult to reconcile with a mean-field lattice gas model. In [16], the coupling to a non-critical mode, specifically a particular Raman-active phonon-mode, was proposed. Raman scattering has so far been conducted only on polycrystalline samples, not finding any indications for this scenario [87]. However, a problem of the model is that with the simple quadratic Hamiltonian (1), an instability at only a single q-position can be ob- 
tained, i.e. the additionally observed reflections near $\left(00 \frac{3}{2}\right)$ cannot be explained.

4.5 Ion-size effects on CO Given the large distortions of the bilayer in $R \mathrm{Fe}_{2} \mathrm{O}_{4}$ as a function of $R$ ionsize (Sec. 2 and Fig. 1b), different CO for different $R$ may be expected. $\mathrm{Yb}$ is quite close in ion size to $\mathrm{Lu}$, and whereas long-range $\mathrm{CO}$ was not yet found, presumably due to stoichiometry-problems, diffuse scattering near $\left(\frac{1}{3} \frac{1}{3} \ell\right)$ is generally observed [95], and an electron-diffraction study [96] even found maxima at $\ell$ half-integer. Given similar spin-charge coupling (see Sec.5.3, the same CO as for $\mathrm{Lu}$ is likely. For $R=\mathrm{In}$, which has a significantly smaller ion size, single crystals are not available, but synchrotron x-ray powder diffraction suggests the appearance of long-range CO below $\sim 250 \mathrm{~K}$, with peaks indexed by $\left(\frac{1}{3} \frac{1}{3} \tau\right)$, with $\tau$ incommensurate [11]: the limit of thick bilayers seem to favor an incommensuration out-of-plane rather than inplane.

For the other extreme of thin bilayers, $R=\mathrm{Y}$, the same type of diffuse scattering along $\left(\frac{1}{3} \frac{1}{3} \ell\right)$ is observed at all $T$ (e.g. [97]) for samples with significant oxygen deficiency as in off-stoichiometric Lu or Yb samples. However, the behavior of sufficiently stoichiometric material is more complex. Abrupt changes of resistivity accompanying the two magnetic transitions marked by arrows in Fig.2 [98] suggest these are also structural transitions, with monoclinic and finally triclinic distortions as indicated by powder x-ray diffraction [99].

Because weak superstructure peaks are difficult to observe in powder diffraction, and sufficiently stoichiometric single crystals were absent until recently, subsequent work on the $\mathrm{CO}$ of stoichiometric $\mathrm{YFe}_{2} \mathrm{O}_{4}$ focused on electron-diffraction (e.g. [97,100,101]). At low $T$, these studies found clear superstructure spots with very different propagation vectors, such as $\left(\frac{1}{14} \frac{2}{7} \frac{1}{14}\right)$ between 85 and $130 \mathrm{~K}$, and consistent with triclinic symmetry. At higher $T$ often a $\left(\frac{1}{4} \frac{1}{4} 0\right)$-type superstructure is observed, and sometimes $\left(\frac{1}{2} \frac{1}{2} 0\right)$ - or more "conventional" $\left(\frac{1}{3} \frac{1}{3} \frac{3}{2}\right)$-types, partly with different phases coexisting or relaxing from one to the other as a function of time. The question of 3D CO in stoichiometric $\mathrm{YFe}_{2} \mathrm{O}_{4}$ above the magnetic ordering temperature, in particular at room temperature, is not clear, with either only diffuse $\left(\frac{1}{3} \frac{1}{3} \ell\right)$ lines reported [100] or with coexistence of diffuse lines with broad $\left(\frac{1}{3} \frac{1}{3} \frac{n}{2}\right)$ spots, the latter by dark-field imaging assessed to correspond to 3D CO domains correlated over some 30 bilayers 97 .

These observations suggest a subtle competition of several $\mathrm{CO}$ phases, that correspond to highly complex charge patterns, in $\mathrm{YFe}_{2} \mathrm{O}_{4}$, reminiscent of some of the predictions based on single-bilayer model Hamiltonians (see Sec. 4.4). To elucidate the strong difference in $\mathrm{CO}$ to $\mathrm{LuFe}_{2} \mathrm{O}_{4}$ will require detailed modeling, but first the real-space $\mathrm{CO}$ giving rise to the observed superstructure reflections will have to be solved, presumably by single-crystal x-ray diffraction on sufficiently stoichiometric crystals [31].

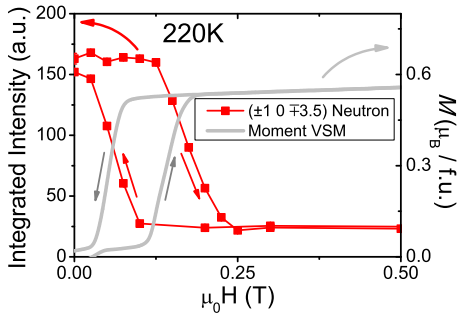

Figure 11 Magnetic field $H$ dependence of magnetization $M$ and the intensity of the $(\overline{1} 02)+\left(00 \frac{3}{2}\right)$ magnetic reflection at $220 \mathrm{~K}$ in $\mathrm{LuFe}_{2} \mathrm{O}_{4}$. After [29].

4.6 Fe-site substitution and intercalation As mentioned in Sec. 2, possible changes in oxygen stoichiometry hamper substitution studies on the $\mathrm{Fe}$ site. Nevertheless, the expected quick depression of $\mathrm{CO}$ or charge diffuse scattering seems to hold (see, e.g., [102]). For intercalated materials, e.g. $\mathrm{Lu}_{2} \mathrm{Fe}_{3} \mathrm{O}_{7}$, superstructure reflections have been observed by electron diffraction, interestingly up to $750 \mathrm{~K}$, and interpreted in terms of both the bilayers and the intercalated single Fe-layers exhibiting CO [38, 103, 104]. X-ray and neutron diffraction on single crystals would certainly be desirable.

5 Magnetism and Spin-Charge coupling The $R \mathrm{Fe}_{2} \mathrm{O}_{4}$ family first attracted attention due to intriguing magnetic properties of Ising spins (pointing perpendicular to the layers) on a strongly frustrated lattice, and the magnetic properties depend strongly on oxygen-stoichiometry (c.f. Sec. 2).

Off-stoichiometric samples typically show significantly higher magnetization when cooled in a magnetic field than when cooled in zero field (c.f. Figs. 2 and 3 a), with a remanent magnetic moment after cooling in strong magnetic fields between $1.5(\mathrm{Y})$ and $2.8 \mu_{B} /$ f.u. (Lu) [105]. Frequency-dependence in ac magnetic susceptibility is observed below about $220-250 \mathrm{~K}$, with detailed analyses suggesting either spin-glass- [106] or clusterglass-like [107, 108, 109] freezing, with large clusters of size $\sim 100 \mathrm{~nm}$ in-plane directly observed by magneticforce-microscopy in one example [107]. Neutron diffraction studies suggest 2D magnetic correlations, with diffuse magnetic scattering typically observed along the $\left(\frac{1}{3} \frac{1}{3} \ell\right)$ line [30, 110] similar to the CO in non-stoichiometric samples (c.f. Sec. 4). This suggests that mainly the correlation between different bilayers is lost. In less off-stoichiometric samples, a tendency towards a development of peaks along this line has been observed [30], but so far few neutron diffraction studies of stoichiometric crystals has been performed, and to date only the magnetic structure of $\mathrm{LuFe}_{2} \mathrm{O}_{4}$ is known, which will therefore be the focus in the following.

5.1 3D magnetic correlations in $\mathrm{LuFe}_{2} \mathrm{O}_{4}$ Sharp magnetic Bragg-reflections have been observed after cooling below $T_{N} \sim 240 \mathrm{~K}$ at $\left(\frac{1}{3}, \frac{1}{3}\right.$,integer) and $\left(\frac{1}{3}, \frac{1}{3}\right.$, halfinteger) positions in several single crystals of $\mathrm{LuFe}_{2} \mathrm{O}_{4}$ by neutron diffraction [29,52,55,56,57] and soft resonant $\mathrm{x}-$ ray diffraction [29]. Magnetic correlations between differ- 


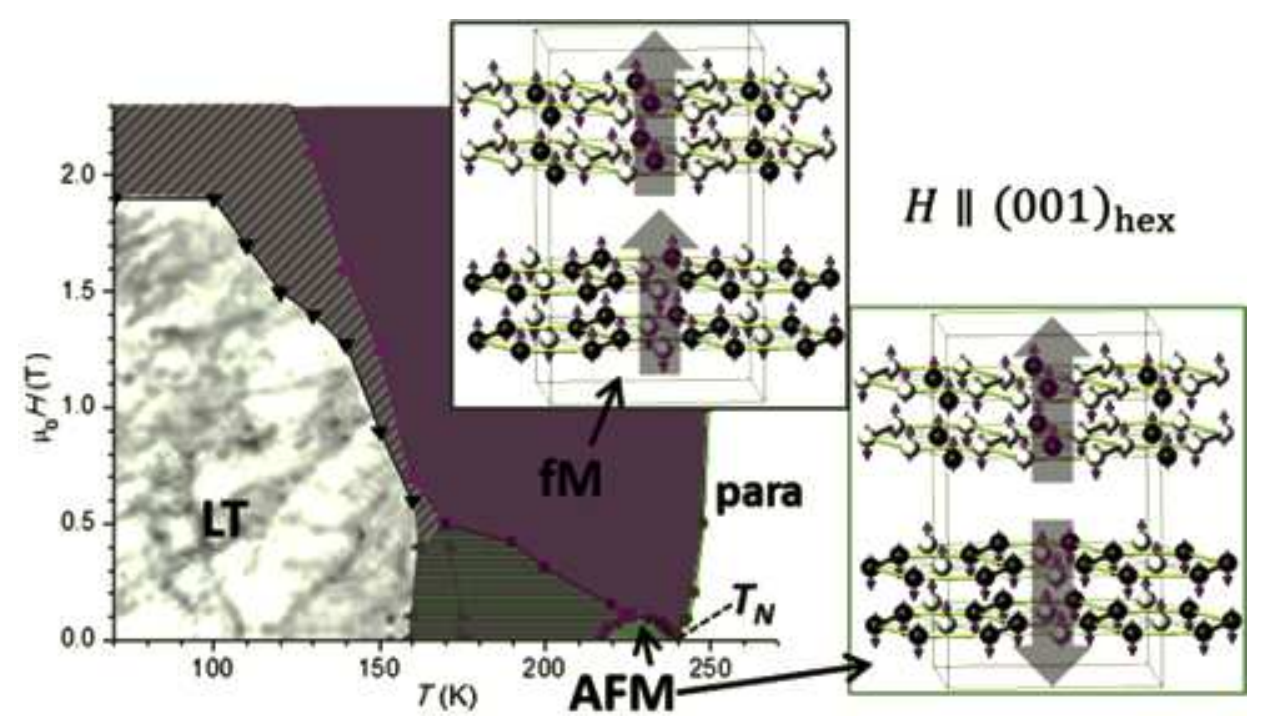

Figure $12 H-T$ Magnetic phase diagram of $\mathrm{LuFe}_{2} \mathrm{O}_{4}$ from data of 2963. Shown are a low- $H$ antiferromagnetic (AFM) and a high- $H$ ferrimagnetic (fM) phase near the ordering temperature $T_{N} \sim 240 \mathrm{~K}$, as well as a lower-temperature phase (LT) with re-entrant disorder [52] and a subtle structural modification [63]. Hysteretic regions where more than one phase can be stabilized are hatched. The $\mathrm{LT} \rightarrow \mathrm{fM}$ transition line $(\triangle)$ reaches $7 \mathrm{~T}$ at $50 \mathrm{~K}$ (not shown) [63]. The spin structures of the AFM and $\mathrm{fM}$ phases [29] are also shown, with large semitransparent arrows indicating the bilayer net magnetizations. ent bilayers have also been deduced from powder neutron diffraction [107,111]. An example of $\left(\frac{1}{3} \frac{1}{3} \ell\right)$ scans at different temperatures is shown in Fig. 7b): the weak reflections at $\left(\frac{1}{3}, \frac{1}{3}\right.$, half-integer) at $280 \mathrm{~K}$ (thick olive) are due to $\mathrm{CO}$, the much larger intensity mainly at $\left(\frac{1}{3}, \frac{1}{3}\right.$, integer) at $220 \mathrm{~K}$ is magnetic in origin, as shown by polarized neutron diffraction (see, e.g. Fig. 5 in [29]), with an out-of-plane correlation length of $15 \mathrm{~nm}$. At $130 \mathrm{~K}$ (red) a large diffuse background appears together with peak-broadening, suggesting a re-entrant magnetic disordering [52,56]. This low-temperature state (LT phase) will be discussed in Sec. 6.2 .

In contrast to stoichiometric $\mathrm{YFe}_{2} \mathrm{O}_{4}$, where the absence of any magnetic-field induced phase transitions is reported [27,99], magnetization-measurements on stoichiometric $\mathrm{LuFe}_{2} \mathrm{O}_{4}$ reveal a complex magnetic field $\left(H \|(001)_{\text {hex }}\right)$-temperature $(T)$ phase diagram [29,63], shown in Fig. 12. Apart from the already mentioned LT phase with reentrant magnetic disorder, and the paramagnetic state, two further phases are present. Isothermal magnetization $M(H)$ loops (e.g. Fig. 11 grey line) are consistent with a hysteretic metamagnetic transition between a low- $H$ phase that is antiferromagnetic (AFM) and a high- $H$ phase that is ferrimagnetic (fM), with a net magnetic moment at low temperatures (the fM phase remains stable down to $T=0$ when cooled in $\mu_{0} H>2 \mathrm{~T}$ ) of $\sim 3 \mu_{B} /$ f.u., suggesting a $\uparrow \uparrow \downarrow$-like arrangement of Fe spins. Partially strong hysteresis of the transition between AFM and fM phases (hatched in Fig. 12), also present between LT and either fM or AFM, is one of the unusual features of the phase diagram. Magnetic Bragg reflections are observed for both the AFM and the fM phase at $\left(\frac{1}{3} \frac{1}{3} \ell\right)$, with an intensity shift surprisingly from $\ell$ integer to halfinteger reflections going from AFM to fM [29,55]. As expected from the presence of a net moment, in the fM phase magnetic intensity is also observed on structural reflections. In contrast, magnetic intensity at $\left(00 \frac{3}{2}\right)$-type reflections is a feature of the AFM phase (see Fig. 11).

5.2 Competing spinstructures Given the correspondence of Ising spins $\uparrow$ or $\downarrow$ with valence states $2+$ and $3+$, both being binary orders, the most straightforward way to analyze spinstructures is to neglect the different spin quantum numbers for the different valence states of the Fe ions and proceed from the hexagonal cell analogous to the $\mathrm{CO}$. As the same propagation vectors are involved as considered for the $\mathrm{CO}$ in Sec. 4.1 there will again be three domains and the same four possible orderings shown in Fig. 6also then apply to the spin order (SO), with black and white coloring now indicating spins $\uparrow$ and $\downarrow$. If the weaker $\left(\frac{1}{3}, \frac{1}{3}\right.$, half-integer) magnetic reflections are considered to be due to a decoration by the $\mathrm{CO}$, then the proper SO should be the ordering shown in Fig. 6 bottom left, because for a $\Gamma_{1}$ representation there would be no intensity on $\left(\frac{1}{3} \frac{1}{3} 0\right)$, which is the strongest magnetic reflection (Fig. 7b). This ferrimagnetic SO was found to fit well all $\left(\frac{1}{3}, \frac{1}{3}\right.$,integer)-type reflections and was thus initially proposed to apply [52].

However, the later found [29] magnetic intensity at $\left(\overline{1} 0 \frac{7}{2}\right.$ ) (Fig. 11) is inconsistent with this SO, showing that symmetry-analysis based on the hexagonal cell cannot describe the $\mathrm{SO}$ of $\mathrm{LuFe}_{2} \mathrm{O}_{4}$. In retrospective this is not surprising, because the SO takes place at $T_{\mathrm{N}}<T_{\mathrm{CO}}$ and if any spin-charge coupling is present (c.f. Sec. 5.3) the proper cell on which $\mathrm{SO}$ is to be considered is the $\mathrm{CO}$ supercell. Because the magnetic cell is equal to the CO cell, symmetry analysis is then based on a (000) propagation, and with four independent sets of Fe sites present in this cell, and four irreducible representations for each site, many SO have to be considered. According to Landau-theory, all sites should order according to the same irreducible representation if the ordering occurs in a second-order phase transition at a single temperature. This condition yields 40 
possible SO, which however were all found to be inconsistent with the experimental data [29]. In a brute-force approach all $3^{12}=531^{\prime} 441 \mathrm{SO}$ of Ising spins $(\uparrow, \downarrow, 0$, allowing for some Fe sites remaining disordered) were compared with the data for both AFM and fM phases, which identified the SO shown in Fig. 12 as the correct ones, verified by refinement [29]. For these SO, all four sets of Fe sites order according to an irreducible representation, but with different ones for different sets, implying that the SO does not occur via a single second-order phase transition. Indeed, given that both AFM and fM, separated by a firstorder transition, are present near $T_{\mathrm{N}}$ and $H=0$, a simple second-order transition seems unlikely.

AFM and fM SO are closely related. In both cases, each bilayer (and each individual layer) has a $2: 1$ ( $\uparrow \uparrow \downarrow$ or $\downarrow \downarrow \uparrow$ ) ferrimagnetic arrangement of the Fe spins, with the bilayer net moment indicated by thick semi-transparent arrows in Fig. 12. For the ferrimagnetic fM phase, the net moments of the two bilayers of the cell are both $\uparrow$, yielding a net moment, as observed. For the AFM phase, the spins in one of the two bilayers are exactly as in the fM phase, and for the other bilayer they are all reversed, yielding the antiferromagnetic behavior indicated by magnetization measurements. Intriguingly, as can be seen in Fig. 12, the AFM-fM transition extrapolates to $H \approx 0$ for $T \rightarrow T_{\mathrm{N}}$, implying the near-degeneracy of these two SO phases at $T_{\mathrm{N}}$ in zero field, a hallmark of geometrical frustration. A competition of AFM and fM SO has also been observed in a study on polycrystalline samples [111]. Because AFM and fM SO are distinct only by the flip of all spins in one of the bilayers, this suggests that the spin correlations within the bilayers are much stronger than the inter-bilayer correlations. In analogy to the $\mathrm{CO}$ (Sec.4.2), a random stacking of the net moments of still relatively well spin-ordered bilayers can then be expected above $T_{\mathrm{N}}$, with diffuse magnetic scattering along $\left(\frac{1}{3} \frac{1}{3} \ell\right)$. This has indeed been observed (Fig. 77), up to room temperature, and deviations of the magnetic susceptibility from Curie-Weiss behavior suggest that spin-fluctuations correlated within each bilayer remain relevant beyond even $T_{\mathrm{CO}}$ [29]. Note that these correlations are dynamic (faster than $\sim \mathrm{MHz}$ ) rather than static, because no indications of magnetic order are visible in Mössbauer spectra at $260 \mathrm{~K}$ [63.

\subsection{Spin-charge coupling}

Experimental indications Experimental indications of a spin-charge coupling by anomalies in the $T$ dependence of the intensity or incommensuration of the $\mathrm{CO}$ superstructure reflections at $T_{N}$ have been observed [44, 58], but they are weak. However, this might be expected if the SO within individual bilayers is formed already above $T_{N}$. The SO refinements [29] yielded some hints of a strict assignment of $\mathrm{SO}$ and $\mathrm{CO}$, but the different magnetic moments of $\mathrm{Fe}^{2+}$ and $\mathrm{Fe}^{3+}$ ions could not be reliably determined. However, spectroscopic techniques performed on a variety of samples provide strong evidence that such spin-charge coupling is a common feature at

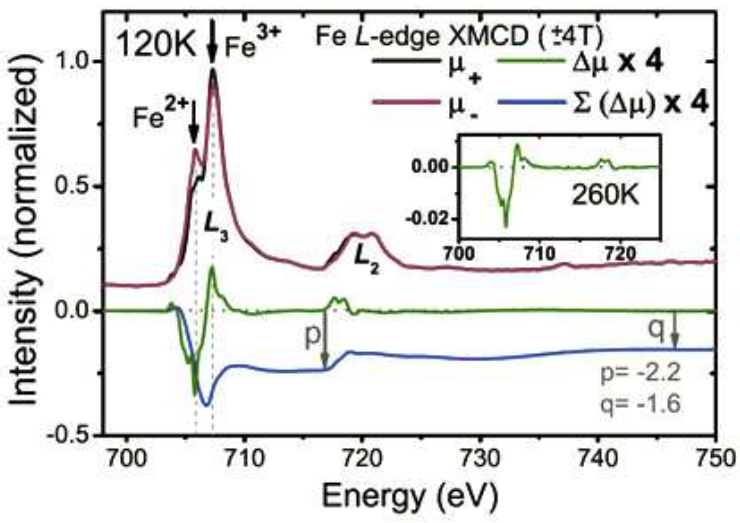

Figure $13 \mathrm{X}$-ray absorption spectra XAS with right $\left(\mu_{+}\right)$ and left $\left(\mu_{-}\right)$circular polarized photons and X-ray Magnetic Circular Dichroism XMCD $\Delta \mu=\mu_{+}-\mu_{-}$of $\mathrm{LuFe}_{2} \mathrm{O}_{4}$ across the $\mathrm{Fe} L_{2 / 3}$ edges in $4 \mathrm{~T}$ (perpendicular to the layers) at $120 \mathrm{~K}$ and $260 \mathrm{~K}$ (inset). Also shown is the integration of the XMCD, with the sum rules yielding an orbital magnetic moment of $\sim 0.7 \mu_{B} /$ f.u.. From [18], (C) 2012 American Physical Society.

least in $\mathrm{YbFe}_{2} \mathrm{O}_{4}$ and $\mathrm{LuFe}_{2} \mathrm{O}_{4}[18,64,112,113,114]$. In the first studies, polarized Mössbauer spectroscopy was applied to single crystals of $\mathrm{YbFe}_{2} \mathrm{O}_{4}\left[113\right.$ and $\mathrm{LuFe}_{2} \mathrm{O}_{4}$ [114] under varying $H$, it was concluded that all of the $\mathrm{Fe}^{2+}$ spins as well as $\frac{1}{3}$ of the $\mathrm{Fe}^{3+}$ spins were aligned $\| H$, with the remaining $\frac{2}{3}$ of the $\mathrm{Fe}^{3+}$ spins aligned antiparallel to $H$.

Later studies by x-ray magnetic circular dichroism (XMCD) confirmed this arrangement for $\mathrm{LuFe}_{2} \mathrm{O}_{4}[18$ 64,112. Fig. 13 shows the absorption spectra and XMCD measured (in the fM-phase) on a crystal of the same type as used to establish the magnetic phase diagram and SO (c.f. Fig. 12). The larger negative peak of the XMCD signal at the $\mathrm{Fe}^{2+} L_{3}$ edge and the smaller positive peak at the $\mathrm{Fe}^{3+} L_{3}$ edge directly implies a larger $\mathrm{Fe}^{2+}$ net moment in magnetic field-direction, and a smaller $\mathrm{Fe}^{3+}$ net moment opposite to this, which given the known magnetic cell-size and saturation magnetization leaves the above spin configuration as the only possible one. From the XMCD sum rules, a sizeable orbital magnetic moment of $\sim 0.7-0.8 \mu_{B} / \mathrm{Fe}^{2+}$ ion can be concluded [29,64, 112], rendering the $\mathrm{Fe}^{2+}$ total magnetic moment to $\sim 4.75 \mu_{B}$, quite close to the spin-only $\mathrm{Fe}^{3+}$ moment of $5 \mu_{B}$. The resulting saturation moment of $4.75-\frac{1}{3} \cdot 5 \sim 3.08 \mu_{B} /$ f.u. is in agreement with magnetization data.

In Fig. 12, both spin and charge structures are shown, and it can be readily seen that for the fM-phase the alignment of $\mathrm{Fe}^{2+}$ and $\mathrm{Fe}^{3+}$ moments according to XMCD and Mössbauer spectroscopy is indeed observed, i.e. the previously discussed $\mathrm{CO}$ and SO are consistent with strict spincharge coupling as deduced spectroscopically. Moreover, the SO shown in Fig. 12 combined with the spectroscopic 


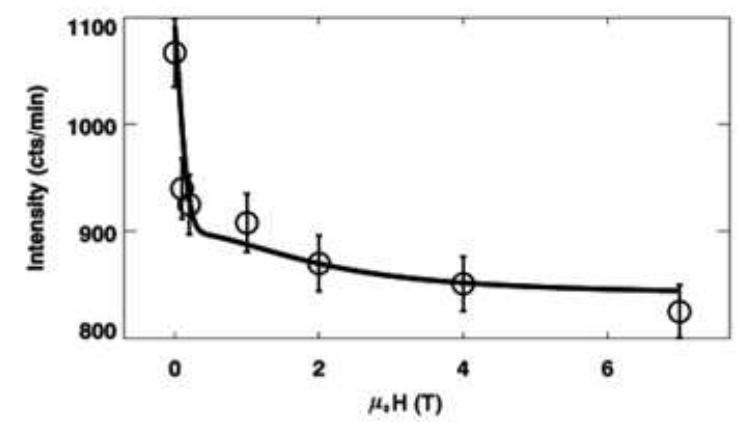

Figure 14 Intensity of the $\left(\frac{2}{3} \frac{2}{3} \frac{7}{2}\right) \mathrm{CO}$ superstructure peak measured by neutron diffraction at $300 \mathrm{~K}$, after cooling in magnetic fields of different strengths. From [55], (c) 2009 American Physical Society.

results is incompatible with any of the $\mathrm{CO}$ shown in Fig. 6 except the charged bilayer stacking deduced in Sec. 4.1 providing additional support for this model of $\mathrm{CO}$ [18].

The inset of Fig. 13 shows a XMCD spectrum measured in $4 \mathrm{~T}$ above $T_{\mathrm{N}}$, at $260 \mathrm{~K}$. Though much reduced in amplitude, the shape is still the same as in the fM-phase below $T_{\mathrm{N}}$. This is consistent with the model of randomlystacked but otherwise still well spin-ordered bilayers in the paramagnetic state: Polarized by a sufficiently strong magnetic field, the random-stacking of $50 \% \uparrow$ and $50 \% \downarrow$ bilayer net moments will be broken, with the overall spin arrangement tending towards the fM SO. It also shows that the spin-charge coupling is very robust, not requiring longrange order. The magnetic correlations could thus be important not only for the magnetic transition at $T_{\mathrm{N}}$, but also for the charge ordering at $T_{\mathrm{CO}}$.

Interestingly, a magnetic-field influence on the $\mathrm{CO}$ has been observed for $\mathrm{LuFe}_{2} \mathrm{O}_{4}$ [55]: Fig. 14] shows the intensity of the $\left(\frac{2}{3} \frac{2}{3} \frac{7}{2}\right) \mathrm{CO}$ superstructure reflection, measured at $300 \mathrm{~K}$, after cooling the crystal in different magnetic fields from $350 \mathrm{~K}$. A significant suppression of $\sim 15 \%$ already by rather small fields is visible. This is surprising, given that at the AFM-fM metamagnetic transition no change of the $\mathrm{CO}$ was observed [18]. However, in contrast to these measurements with $H \| c$, in [55] the field was applied in [110] direction. This in-plane $H$ breaks the three-fold rotation symmetry of the crystal, and likely affects the relative stability of the three magnetic domains (based, as the CO domains, on the three monoclinic cells shown in Fig. 11). The strict spin-charge coupling implies the correspondence of magnetic and CO domains, and therefore an in-plane $H$ should affect the domain population. The $\left(\frac{2}{3} \frac{2}{3} \frac{7}{2}\right)$ reflection studied in [55] belongs to one of the CO domains, its intensity suppression by $H$ suggests that population of other $\mathrm{CO}$ domains is favored, which could be tested by simultaneously tracking different $\mathrm{CO}$ reflections belonging to all domains.

Theoretical considerations The single-bilayer lattice gas models [90,91,92,93,94] also consider differ- ent SO and spin-charge coupling, finding that the spincorrelations tend to stabilize CO with $\left(\frac{1}{3} \frac{1}{3} \ell\right)$-type propagation compared to alternatives (c.f. Sec. 4.4). However, because these models do not consider the charged bilayers indicated by experiment, the focus below is on more phenomenological considerations and the DFT calculation [89].

Because the inter-bilayer spin-coupling is very weak (c.f. Sec. 5.2), it is a good first approximation to consider each bilayer, the one with $\mathrm{Fe}^{2+}$ majority and the one with $\mathrm{Fe}^{3+}$ majority, individually. Various magnetic superexchange parameters between Fe ions of the same layer and between the two layers have then to be examined, which may be expected to strongly depend on the valence state of the respective $\mathrm{Fe}$ ions. An experimental determination of exchange parameters could be done by inelastic neutron scattering, which so far have been reported only in a limited range on a crystal [78], and on a polycrystalline sample, with the observation of a spin-gap $\sim 7 \mathrm{meV}$ confirming the Ising nature of spins [111].

The exchange parameters were estimated theoretically by mapping the energy differences between differently ordered states obtained by DFT to the corresponding energy differences of an Ising Hamiltonian [89]. This study considered an individual bilayer, however, one assumed to exhibit the ferroelectric CO (Fig. 6 top left). The by far largest interaction was found to be an antiferromagnetic superexchange between $\mathrm{Fe}^{3+}$ ions of the same $\mathrm{Fe}^{3+}$ majority layer. From this, an in-plane antiferromagnetic arrangement of $\mathrm{Fe}^{3+}$ moments is expected, which is indeed observed experimentally (see Fig. 12). A corresponding calculation taking into account the charged bilayer $\mathrm{CO}$ would be highly desirable: as mentioned already in Sec. 4.4, the large energy-differences between different spinstates found in [89] imply that the $\mathrm{CO}$ and SO have to be calculated together, in a cell of sufficient size, which makes the calculations challenging.

5.4 Off-stoichiometric samples and other rare earths The agreement between XMCD experiments conducted on samples with [18] and without [64] long-range $\mathrm{CO}$ indicates that the strict spin-charge coupling with the $\mathrm{Fe}^{2+} \uparrow \uparrow \uparrow / \mathrm{Fe}^{3+} \uparrow \downarrow \downarrow$ arrangement is a robust and generic feature of $\mathrm{LuFe}_{2} \mathrm{O}_{4}$. Because the superexchange interactions leading to spin-charge coupling and SO depend on the underlying $\mathrm{CO}$, it seems very likely that in all samples individual bilayers exhibit both $\mathrm{CO}$ and $\mathrm{SO}$ as discussed in Secs. 4.1, 4.2 and 5.2 with only the occurrence of long-range $3 \mathrm{D}$ order with correlations between different bilayers being affected by stoichiometry. The generic observation of at least diffuse charge (e.g. [107]) and diffuse magnetic (e.g. [110]) scattering at the $\left(\frac{1}{3} \frac{1}{3} \ell\right)$ line supports this assessment. Because the charge dynamics slows down upon cooling regardless of stoichiometry [25], a glassy freezing of the essentially still charge-ordered bilayers can be expected to replace the long-range $\mathrm{CO}$ when the latter is prevented. Similarly, a glassy freezing of SO bi- 
layers can be expected to replace the long-range AFM phase upon cooling, and such a cluster-glass-like behavior involving frequency-dependence in ac susceptibility has indeed been found for some samples, e.g. in [108]. On the other hand, when a magnetic field forces the net moments of all bilayers to align, a stable fM-like phase with the same saturation moment should be recovered, as is indeed suggested by all macroscopic measurements (see e.g. [106 108] for corresponding phase diagrams) and by electron holography [115]. Note, however, that because of the underlying random stacking of $\mathrm{Fe}^{2+}$ and $\mathrm{Fe}^{3+}$ majority bilayers, this fM phase is not expected to be 3D ordered, but should rather exhibit diffuse magnetic scattering along $\left(\frac{1}{3} \frac{1}{3} \ell\right)$, which could be checked by in-field neutron diffraction on less-stoichiometric crystals. Thus, based on the above, for off-stoichiometric samples, exactly the "spinglass-like 2D-ferrimagnetic order" proposed early on [110] is expected to occur.

The above considerations should hold also for $\mathrm{YbFe}_{2} \mathrm{O}_{4}$ which has a similar $R$ ion size. Indeed, not only the same diffuse scattering at $\left(\frac{1}{3} \frac{1}{3} \ell\right)$ has been observed [95] as on non-stoichiometric $\mathrm{LuFe}_{2} \mathrm{O}_{4}$, but the same spin-charge coupling is also deduced [113]. For $R=$ In with considerably smaller ion size, magnetism and spin-charge coupling appear to be rather different as indicated both by a much smaller saturation moment of $1.5 \mu_{B} /$ f.u. and dissimilar Mössbauer spectra [11]. An XMCD study on $\mathrm{InFe}_{2} \mathrm{O}_{4}$ might be helpful to elucidate spin-charge coupling in this material. For $R$ with considerably larger ionic radii than $\mathrm{Lu}$ or $\mathrm{Yb}$, there are also clear differences. For example, in stoichiometric $\mathrm{YFe}_{2} \mathrm{O}_{4}$ there is no $\mathrm{fM}$ phase, and given the wildly varying $\mathrm{CO}$ observed at low $T$ (see Sec. 4.6) a similar spin-charge coupling as in $\mathrm{LuFe}_{2} \mathrm{O}_{4}$ is rather doubtful, despite similarities of diffuse scattering observed in off-stoichiometric crystals. In agreement with substantial changes upon increasing the rare earth ionic size, an XMCD study on $\mathrm{LuFe}_{2} \mathrm{O}_{4}$ with $\mathrm{Lu}$ partially substituted by the larger Y or Er ions [116], found marked changes in the spectra. Whereas the negative peak at the $\mathrm{Fe}^{2+}$ edge remained largely unaffected by the substitution, suggesting that "all $\mathrm{Fe}^{2+}$ spins aligned with $H$ " still holds, the positive peak at the $\mathrm{Fe}^{3+}$ edge was completely suppressed for $\sim 50 \%$ substitution, suggesting an antiferromagnetic order of $\mathrm{Fe}^{3+}$ spins.

\section{The ground state: orbital order?}

6.1 Orbital order, orbital magnetic momentum, and magnetic anisotropy Concomitant with $\mathrm{CO}$ and $\mathrm{SO}$, orbital order (OO) typically occurs in other transition metal oxides, strongly influencing the magnetism as well [117. The possible presence of $\mathrm{OO}$ has been considered for $\mathrm{LuFe}_{2} \mathrm{O}_{4}$ theoretically [84,90,92], for example the DFT calculations [84] suggest a clear OO. However, the discrepancy of the predicted $\mathrm{CO}$ with the experimentally deduced one suggests that the predictions of $\mathrm{OO}$ also have to be taken with care.

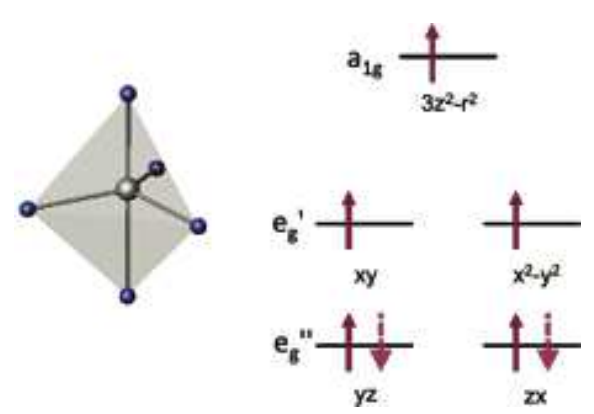

Figure 15 Sketch of the energy-splitting of the $\mathrm{Fe} 3 d$ orbitals (right) due to the trigonal-bipyramidal coordination (left). For $\mathrm{Fe}^{3+}$, each orbital is singly occupied, the extraelectron of $\mathrm{Fe}^{2+}$ can go to either of two $e_{g}^{\prime \prime}$ orbitals.

The natural starting point for the consideration of $\mathrm{OO}$ is the trigonal bipyramidal crystal-field level diagram shown in Fig. 15. For $\mathrm{Fe}^{3+}$, all five orbitals are singly occupied by a spin $\uparrow$ electron, and there is no orbital degree of freedom. However, the extra $\downarrow$-electron at $\mathrm{Fe}^{2+}$ ions can go to an orbital of the doubly-degenerate lowest level. It should be mentioned that $e_{g}^{\prime}$ and $e_{g}^{\prime \prime}$ levels are quite close in energy in $\mathrm{LuFe}_{2} \mathrm{O}_{4}$ : while band-structure calculations suggested that $e_{g}^{\prime}$ is the lowest level [84], polarized x-ray absorption spectroscopy at the $\mathrm{O} K$-edge has later shown that the $e_{g}^{\prime \prime}$ level is actually lower [64].

OO would then imply a preferential occupation of either the $y z$ or the $z x$ orbital - or a given real linear combination of these - for each site. An alternative to this is the preferential occupation of a complex linear combination that could carry an orbital angular momentum $\mathbf{L}(\langle\psi|\mathbf{L}| \psi\rangle=0$ for $\psi$ real $)$ and thus an orbital magnetic moment. From a linear combination of $y z$ and $z x$ orbitals, no eigenstates of $L_{x}$ or $L_{y}$ can be obtained, but $\sqrt{\frac{1}{2}}(\mp z x-i \cdot y z)$ is an eigenstate of $L_{z}$ with eigenvalue $m= \pm 1$, allowing an orbital magnetic moment of up to $\pm 1 \mu_{B} / \mathrm{Fe}^{2+}$ ion. The fact that an orbital magnetic moment $\sim 0.7-0.8 \mu_{B} / \mathrm{Fe}^{2+}$ is observed (see Sec.5.3) implies that in the $\mathrm{fM}$ state (and at low $T$ for samples without longrange $\mathrm{SO}$ ) $\mathrm{OO}$ cannot occur. Consistent with this, no additional reflections have ever been found, and no anisotropies in resonant diffraction suggestive of ferro-type OO have been found [42]. For samples with long-range SO, this conclusion can also be extended to the AFM and paramagnetic phases (c.f. Fig. 12), because high-resolution x-ray diffraction indicates no structural changes that necessarily would accompany an OO-transition at the fM-AFM or AFM-paramagnetic transitions [18] (though for the latter small intensity anomalies are suggested in [44,58]).

From the presence of an orbital magnetic moment necessarily perpendicular to the layers, the large magnetic anisotropy making this a model Ising-system follows immediately, because spin-orbit coupling aligns the directions of spin- and orbital magnetic moments [64]. The large 
magnetic anisotropy is to a good part responsible for the giant coercivity of $\mathrm{LuFe}_{2} \mathrm{O}_{4}$, with coercive fields reaching around $10 \mathrm{~T}$ at low $T$ [23,107], and to "exchange-bias"like properties [118].

6.2 The low-temperature phase The only phase in the $H-T$ phase diagram (Fig. 12) consistent with $\mathrm{OO}$ is the LT-phase, because both AFM $\leftrightarrow$ LT and fM $\leftrightarrow$ LT transitions are also structural transitions as indicated by Bragg-peak splitting [63]. As discussed in Sec. 4.2, the transition to this phase is accompanied by the complete freezing of residual charge dynamics, which is consistent with an $\mathrm{OO}$ scenario, given that $\mathrm{OO}$ often accompanies metal-insulator transitions [117]. Due to the impact of OO on the magnetic exchange interactions, a change of the magnetic structure upon entering the LT phase would then be expected, consistent with the observed re-entrant disorder (Fig. 7b).

These indications that $\mathrm{OO}$ might be the driving force behind the transition into the LT phase are, however, at most tentative. As discussed in Sec. 6.1, a full OO would imply the quenching of the orbital magnetic moment and the removal of the strong tendency of spins to be aligned perpendicular to the layers. The relatively good description of the $\ell$-dependence of the diffuse component of magnetic scattering at $130 \mathrm{~K}$ assuming spin direction perpendicular to the layers [52] is therefore an argument against an OO scenario.

Structurally, despite of the Bragg-peak splitting, there is no basic change of the $\mathrm{CO}$ across $T_{\mathrm{LT}}$ : the interbilayer correlations of CO seem to improve slightly [44], but the cell size (no extra reflections), $C 2 / m$ symmetry, and the valence states of the Fe ions estimated by bond-valencesum are unaffected within error bars [18]. Although small changes in lattice parameters have been reported, the changes of refined atom positions are much too subtle to conclude about a possible OO [18]. Further structural refinement studies across $T_{\mathrm{LT}}$ are certainly desirable. A further possibility to detect $\mathrm{OO}$ is to assess asymmetries in resonant $\mathrm{x}$-ray diffraction, ideally at the $\mathrm{Fe} L$ edges at the $\left(\tau \tau \frac{3}{2}\right)$ reflections [82,119], although the analysis is complicated by the possible emergence of magnetic intensity at these positions in the LT phase [52,56].

The nature of the LT phase, and whether it is connected with $\mathrm{OO}$ therefore is an open question left for future work. Its experimental investigation is also hampered by its very fragile nature, as it is present in some samples showing long-range $\mathrm{CO}$ and SO [18, 29,52,56, 63] (indications are also visible in $M(T)$ on the polycrystalline samples studied in [22,111]), but not in others [15,55]. Due to this, it is also not completely clear whether the LT phase represents the true ground state of $\mathrm{LuFe}_{2} \mathrm{O}_{4}$, though it appears likely. If the LT phase is the true ground state, it will make theoretical investigations with zero-temperature methods, such as DFT, difficult because of the presence of significant magnetic disorder.
7 Summary and outlook In the following, I will summarize the main results reviewed and highlight critical points for future investigations on rare earth ferrites, concluding by answering the title question and by looking at the larger picture of possible CO-based ferroelectricity in other compounds.

For the most studied rare earth ferrite, $\mathrm{LuFe}_{2} \mathrm{O}_{4}$, a consistent and rather comprehensive picture of strongly coupled charge- and spinorder has emerged from experimental work. Single-crystal x-ray and neutron diffraction revealed sharp charge order (CO) superstructure peaks below $T_{\mathrm{CO}} \sim 320 \mathrm{~K}$ and spin order (SO) superstructure peaks below $T_{\mathrm{N}} \sim 240 \mathrm{~K}$ in stoichiometric samples. Both are unaffected by static electric fields or currents, except for selfheating effects. The corresponding orders have been determined by refinements. The $\mathrm{CO}$ involves charged, rather than polar, bilayers. As revealed by Mössbauer and optical spectroscopies, substantial charge fluctuations occur even below $T_{\mathrm{CO}}$, down to $T_{\mathrm{LT}} \sim 170 \mathrm{~K}$. Between $T_{\mathrm{LT}}$ and $T_{\mathrm{N}}$ two related SO phases compete, due to geometrical frustration. Below $T_{\mathrm{LT}}$, a subtle structural distortion and reentrant magnetic disorder occur. Whether this is connected with possible orbital ordering is a major question left for future work. Mössbauer spectroscopy and x-ray magnetic circular dichroism demonstrate a strict coupling of SO and $\mathrm{CO}$, present also above $T_{\mathrm{N}}$ and likely even above $T_{\mathrm{CO}}$, where diffuse scattering experiments indicate that within individual bilayers $\mathrm{SO}$ and $\mathrm{CO}$ correlations are still strong. The presence of coupled SO and CO correlations within individual bilayers is a robust feature of the compound. However, oxygen off-stoichiometry quickly destroys the interbilayer spin- and charge-correlations, leading to "glassy" behavior.

While charge and spin order have been established, they are not really understood: this would imply elucidating what drives these orders, whereas currently no theoretical works have considered the experimental charge or spin order. What is clear is that the charge order cannot be driven entirely or even predominantly by electrostatic repulsion, because the realized pattern is not the one minimizing this repulsion. Lattice effects as well as magnetic exchange appear to be relevant, but the determination of the main driving forces remains a challenge principally for future theoretical work, though experimental work on phonons and magnetic excitations could make an important contribution: this is the most critical issue for future investigations.

Rare earth ferrites other than $\mathrm{LuFe}_{2} \mathrm{O}_{4}$ have been studied much less, which is largely due to the absence of single crystals that are stoichiometric enough to reveal 3D CO and SO. With this hurdle being overcome [31], progress can be expected. $\mathrm{CO}$ and $\mathrm{SO}$ correlations leading to diffuse scattering very similar as in off-stoichiometric $\mathrm{LuFe}_{2} \mathrm{O}_{4}$ are commonly observed, and for $\mathrm{YbFe}_{2} \mathrm{O}_{4}$ also Mössbauer spectroscopy leads to the expectation of overall behavior very similar to $\mathrm{LuFe}_{2} \mathrm{O}_{4}$. However, for rare earths (or 
In) with larger deviations of ionic size from Lu, experiments on high-quality polycrystalline samples indicate that completely different $\mathrm{CO}$ and $\mathrm{SO}$ is established. For example, electron diffraction on $\mathrm{YFe}_{2} \mathrm{O}_{4}$ demonstrates a number of competing very complex $C O$ phases that have yet to be solved. A partial alternative to single-crystal work is to rely on experimental techniques not requiring longrange $\mathrm{CO}$ and $\mathrm{SO}$, such as $\mathrm{XMCD}$, which has been successfully used to study ion-size effects on spin-charge coupling [116]. The intercalated compounds $\mathrm{R}_{2} \mathrm{Fe}_{3} \mathrm{O}_{7}$ have been even less studied, but given indications of charge order stable up to much higher temperatures [103] deserve more attention. A similar tuning as with intercalation may be possible with thin films [120,121,122, 123], on which, however, $3 D$ charge ordering has yet to be demonstrated.

Ferroelectricity from charge ordering? While the coupled complex charge and spin orders on a geometrically frustrated lattice are also of high intrinsic interest, it is not the property that has attracted most research to the rare earth ferrites. The title question "Ferroelectricity from iron valence ordering in rare earth ferrites?" according to current experimental knowledge has to be answered in the negative, at least for the "prototype" $\mathrm{LuFe}_{2} \mathrm{O}_{4}$. The charge order leading to the $\left(\frac{1}{3} \frac{1}{3} \ell\right)$-type reflections does not involve polar bilayers, and according to recent dielectric spectroscopy studies, the large remanent polarization deduced from pyroelectric current measurements may likely be affected by contact effects. Future theoretical work leading to an understanding of what drives the experimentally indicated non-polar charge order would be important for a final resolution and to provide hints for other families of compounds. In rare earth ferrites other than $\mathrm{LuFe}_{2} \mathrm{O}_{4}$ the charge order can be different and has not been solved, therefore in principle the possibility of ferroelectricity from charge ordering remains within the family. However, with dielectric behavior quite common among other $R \mathrm{Fe}_{2} \mathrm{O}_{4}$ and even the most likely ferroelectric behavior in a doped intercalated compound suggesting a polarization three orders of magnitude weaker than what is expected for ferroelectricity originating from charge ordering, there are no strong indications for success.

Looking beyond rare earth ferrites, the findings reviewed here provide some general lessons for $\mathrm{CO}$-induced ferroelectricity. First and most important, a non-negligible residual conductivity is unavoidable in $\mathrm{CO}$ materials, particularly at and above $T_{\mathrm{CO}}$. Apart from being detrimental with regards to applications, this also renders the standard macroscopic probes of ferroelectricity doubtful as pointed out in [45] and for rare earth ferrites discussed in Sec. 3. Therefore it is imperative to obtain also microscopic proof of at least a polar crystal structure, better yet of a polar crystal structure that can be switched by an electric field - though for such in-electric field measurements residual conductivity and associated self-heating effects are also problematic, see Sec. 4.3. Second, CO materials have a propensity for variable stoichiometry, which can make the elucidation of the CO crystal structure very difficult. Therefore, paying due respect to sample quality is crucial. Finally, in CO materials there is often a strong coupling of charge with orbital and spin degrees of freedom leading to complexity that hampers theoretical treatments. For rare earth ferrites, theoretical work suggests and/or assumes ferroelectric CO (see Sec. 4.4), in contrast to the experimental findings. For other materials, there are many more examples of compounds proposed to exhibit ferroelectricity from $\mathrm{CO}$ based on calculations than examples of experimental indications of the same.

With the prototypical example of rare earth ferrites apparently being a non-example, a valid "proof-of-principle" example of a material exhibiting multiferroicity driven by charge ordering has yet to be demonstrated experimentally. Among the few possibilities in oxides with at least some experimental indications most promising seems classical magnetite, which does seem to have a charge-ordered crystal structure that is polar [124], although it appears to be of a relaxor-type with switching only possible at low $T$ and high frequency [125]. Apart from oxides, low-dimensional molecular compounds (see e.g. [126]) are increasingly being scrutinized for multiferroicity originating from CO. A recently proposed [127], but also disputed [128], example is a metal-organic charge-transfer salt with apparent small polarization but no demonstrated polar crystal structure. Although the temperature scale in all of the above is far below room temperature, an unambiguous proof of the mechanism would be important, opening the route to search for such materials closer to applicability.

Acknowledgements I thank J. de Groot, T. Mueller, R. P. Hermann, and in particular Th. Brückel, for useful comments on draft versions of this article, T. Müller also for digitizing the data from [27] shown in Fig.2 I am very grateful to my students and to the many collaborators I had while working in this field, in particular the coauthors of Refs. [18, 19,7 7282944 52 63 108]. Special thanks go to D. Mandrus for first suggesting this field to me, to M. Subramanian for providing high-quality $\mathrm{LuFe}_{2} \mathrm{O}_{4}$ powder samples very early-on, and to J. de Groot for his immense contributions during his $\mathrm{PhD}$ studies. Finally, I would like to acknowledge financial support from the initiative and networking fund of the Helmholtz Association by funding the Helmholtz-University Young Investigator Group VH NG-510 "Complex Ordering Phenomena in Multifunctional Oxides".

\section{References}

[1] M. Fiebig and N. A. Spaldin, Eur. Phys. J. B 71, 293 (2009).

[2] M. Bibes and A. Barthélémy, Nat. Mater. 7, 425 (2008).

[3] N. A. Hill, J. Phys. Chem. B 104, 6694 (2000).

[4] K. F. Wang, J. M. Liu, and Z. F. Ren, Adv. Physics 58, 321 (2009).

[5] J. van den Brink and D. I. Khomskii, J. Phys.: Condens. Matter 20, 434217 (2008).

[6] Y. Sun, L. Yan, and J. Cong, Sci. China Phys., Mech. and Astron. 56, 222 (2013). 
[7] X. S. Xu, J. de Groot, Q. C. Sun, B. C. Sales, D. Mandrus, M. Angst, A. P. Litvinchuk, and J. L. Musfeldt, Phys. Rev. B 82, 014304 (2010).

[8] K. Kato, I. Kawada, N. Kimizuka, and T. Katsura, Z. Kristallogr. 141, 314 (1975).

[9] M. Isobe, N. Kimizuka, J. Iida, and S. Takekawa, Acta Cryst. C 46, 1917 (1990).

[10] T. Matsumoto, N. Mori, J. Iida, M. Tanaka, K. Siratori, F. Izumi, and H. Asano, Physica B 180, 603 (1992).

[11] K. Oka, M. Azuma, N. Hayashi, S. Muranaka, Y. Narumi, K. Kindo, S. Ayukawa, M. Kato, Y. Koike, Y. Shimakawa, and M. Takano, J. Phys. Soc. Jpn. 77, 064803 (2008).

[12] K. Yoshii, N. Ikeda, and A. Nakamura, Physica B 378380, 585 (2006).

[13] K. Yoshii, Y. Yoneda, D. Maeda, Y. Yokota, T. Michiuchi, T. Komatsu, N. Ikeda, Y. Matsuo, and S. Mori, Jpn. J. Appl. Phys. 47, 7599 (2008).

[14] K. Yoshii, N. Ikeda, Y. Okajima, Y. Yoneda, Y. Matsuo, Y. Horibe, and S. Mori, Inorg. Chem. 47, 6493 (2008).

[15] Y. Yamada, K. Kitsuda, S. Nohdo, and N. Ikeda, Phys. Rev. B 62, 12167 (2000).

[16] A. B. Harris and T. Yildirim, Phys. Rev. B 81, 134417 (2010).

[17] N. Ikeda, H. Ohsumi, K. Ohwada, K. Ishii, T. Inami, K. Kakurai, Y. Murakami, K. Yoshii, S. Mori, Y. Horibe, and H. Kito, Nature 436, 1136 (2005).

[18] J. de Groot, T. Mueller, R. A. Rosenberg, D. J. Keavney, Z. Islam, J. W. Kim, and M. Angst, Phys. Rev. Lett. 108, 187601 (2012).

[19] D. Niermann, F. Waschkowski, J. de Groot, M. Angst, and J. Hemberger, Phys. Rev. Lett. 109, 016405 (2012).

[20] A. Ruff, S. Krohns, F. Schrettle, V. Tsurkan, P. Lunkenheimer, and A. Loidl, Eur. Phys. J. B 85, 290 (2012).

[21] N. Kimizuka and T. Katsura, J. Solid State Chem. 13, 176 (1975).

[22] J. Iida, Y. Nakagawa, and N. Kimizuka, J. Phys. Soc. Jpn. 55, 1434 (1986).

[23] J. Iida, Y. Nakagawa, S. Takekawa, and N. Kimizuka, J. Phys. Soc. Jpn. 56, 3746 (1987).

[24] T. Sugihara, K. Siratori, I. Shindo, and T. Katsura, J. Phys. Soc. Jpn. 45, 1191 (1978)

[25] M. Tanaka, K. Siratori, and N. Kimizuka, J. Phys. Soc. Jpn. 53, 760 (1984).

[26] R. Moessner and A. P. Ramirez, Phys. Today 59(2), 24 (2006).

[27] M. Inazumi, Y. Nakagawa, M. Tanaka, N. Kimizuka, and K. Siratori, J. Phys. Soc. Jpn. 50, 438 (1981).

[28] M. H. Phan, N. A. Frey, H. Srikanth, M. Angst, B.C. Sales, and D. Mandrus, J. Appl. Phys. 105, 07E308 (2009).

[29] J. de Groot, K. Marty, M. D. Lumsden, A. D. Christianson, S.E. Nagler, S. Adiga, W. J.H. Borghols, K. Schmalzl, Z. Yamani, S.R. Bland, R. de Souza, U. Staub, W. Schweika, Y. Su, and M. Angst, Phys. Rev. Lett. 108, 037206 (2012).

[30] S. Funahashi, J. Akimitsu, K. Siratori, N. Kimizuka, M. Tanaka, and H. Fujishita, J. Phys. Soc. Jpn. 53, 2688 (1984).

[31] T. Mueller, Diploma Thesis, RWTH Aachen University (2012).
[32] J. Iida, M. Tanaka, H. Kito, and J. Akimitsu, J. Phys. Soc. Jpn. 59, 4190 (1990).

[33] F. Wang, J. Kim, G. D. Gu, Y. Lee, S. Bae, and Y. J. Kim, J. Appl. Phys. 113, 063909 (2013).

[34] T. Michiuchi, Y. Yokota, T. Komatsu, H. Hayakawa, T. Kuroda, D. Maeda, Y. Matsuo, S. Mori, K. Yoshii, N. Hanasaki, T. Kambe, and N. Ikeda, Ferroelectrics 378, 175 (2009).

[35] K. Yoshii, N. Ikeda, Y. Matsuo, Y. Horibe, and S. Mori, Phys. Rev. B 76, 024423 (2007).

[36] Y. Hou, Y. P. Yao, S. N. Dong, X. Huang, X. Sun, and X. Li, J. Mater. Res. 27, 922 (2012).

[37] K. Kato, I. Kawada, N. Kimizuka, I. Shindo, and T. Katsura, Z. Kristallogr. 143, 278 (1976).

[38] Y. B. Qin, H. X. Yang, Y. Zhang, H. F. Tian, C. Ma, L. J. Zeng, and J. Q. Li, Appl. Phys. Lett. 95, 072901 (2009).

[39] J. Rouquette, J. Haines, A. Al-Zein, P. Papet, F. Damay, J. Bourgeois, T. Hammouda, F. Doré, A. Maignan, M. Hervieu, and C. Martin, Phys. Rev. Lett. 105, 237203 (2010).

[40] N. Ikeda, K. Kohn, H. Kito, J. Akimitsu, and K. Siratori, J. Phys. Soc. Jpn. 63, 4556 (1994).

[41] M. A. Subramanian, T. He, J.Z. Chen, N. S. Rogado, T. G. Calvarese, and A. W. Sleight, Adv. Mater. 18, 1737 (2006).

[42] A. M. Mulders, S. M. Lawrence, U. Staub, M. GarciaFernandez, V. Scagnoli, C. Mazzoli, E. Pomjakushina, K. Conder, and Y. Wang, Phys. Rev. Lett. 103, 077602 (2009).

[43] Y. Yamada, S. Nohdo, and N. Ikeda, J. Phys. Soc. Jpn. 66, 3733 (1997).

[44] M. Angst, R. P. Hermann, A. D. Christianson, M. D. Lumsden, C. Lee, M. H. Whangbo, J. W. Kim, P. J. Ryan, S. E. Nagler, W. Tian, R. Jin, B. C. Sales, and D. Mandrus, Phys. Rev. Lett. 101, 227601 (2008).

[45] M. Maglione and M. A. Subramanian, Appl. Phys. Lett. 93, 032902 (2008).

[46] J. Y. Park, J. H. Park, Y. K. Jeong, and H. M. Jang, Appl. Phys. Lett. 91, 152903 (2007).

[47] D. S. F. Viana, R. A. M. Gotardo, L. F. Cótica, I. A. Santos, M. Olzon-Dionysio, S.D. Souza, D. Garcia, J. A. Eiras, and A. A. Coelho, J. Appl. Phys. 110, 034108 (2011).

[48] J. F. Scott, J. Phys.: Condens. Matter 20, 021001 (2008).

[49] P. Ren, Z. Yang, W. G. Zhu, C. H. A. Huan, and L. Wang, J. Appl. Phys. 109, 074109 (2011).

[50] T. Kambe, Y. Fukada, J. Kano, T. Nagata, H. Okazaki, T. Yokoya, S. Wakimoto, K. Kakurai, and N. Ikeda, Phys. Rev. Lett. 110, 117602 (2013).

[51] Y. Chen, J. Dai, K. Au, K. Lam, Y. Qin, and H. Yang, Materials Lett. 68, 54 (2012).

[52] A. D. Christianson, M. D. Lumsden, M. Angst, Z. Yamani, W. Tian, R. Jin, E. A. Payzant, S. E. Nagler, B. C. Sales, and D. Mandrus, Phys. Rev. Lett. 100, 107601 (2008).

[53] S. Park, Y. Horibe, Y. J. Choi, C. L. Zhang, S. W. Cheong, and W. Wu, Phys. Rev. B 79, 180401 (2009).

[54] N. Ikeda, J. Phys.: Condens. Matter 20(4), 434218 (2008).

[55] J. Wen, G. Xu, G. Gu, and S. M. Shapiro, Phys. Rev. B 80, 020403 (2009). 
[56] J. Wen, G. Xu, G. Gu, and S. M. Shapiro, Phys. Rev. B 81, 144121 (2010).

[57] A. M. Mulders, M. Bartkowiak, J. R. Hester, E. Pomjakushina, and K. Conder, Phys. Rev. B 84, 140403 (2011).

[58] M. Bartkowiak, A. M. Mulders, V. Scagnoli, U. Staub, E. Pomjakushina, and K. Conder, Phys. Rev. B 86, 035121 (2012).

[59] J. Bourgeois, M. Hervieu, M. Poienar, A. M. Abakumov, E. Elkaïm, M. T. Sougrati, F. Porcher, F. Damay, J. Rouquette, G. Van Tendeloo, A. Maignan, J. Haines, and C. Martin, Phys. Rev. B 85, 064102 (2012).

[60] Y. Zhang, H.X. Yang, C. Ma, H.F. Tian, and J. Q. Li, Phys. Rev. Lett. 98, 247602 (2007).

[61] Y. Zhang, H. X. Yang, Y. Q. Guo, C. Ma, H. F. Tian, J. L. Luo, and J. Q. Li, Phys. Rev. B 76, 184105 (2007).

[62] H. Yang, H. Tian, Y. Zhang, Y. Qin, L. Zeng, C. Ma, H. Shi, and J. Lu, Solid State Comm. 150, 1467 (2010).

[63] X. S. Xu, M. Angst, T. V. Brinzari, R. P. Hermann, J. L. Musfeldt, A.D. Christianson, D. Mandrus, B.C. Sales, S. McGill, J. W. Kim, and Z. Islam, Phys. Rev. Lett. 101, 227602 (2008).

[64] K. T. Ko, H. J. Noh, J. Y. Kim, B. G. Park, J. H. Park, A. Tanaka, S. B. Kim, C. L. Zhang, and S.W. Cheong, Phys. Rev. Lett. 103, 207202 (2009).

[65] M. Angst, R. P. Hermann, W. Schweika, J. W. Kim, P. Khalifah, H.J. Xiang, M. H. Whangbo, D. H. Kim, B. C. Sales, and D. Mandrus, Phys. Rev. Lett. 99, 256402 (2007).

[66] E. F. Bertaut, Acta Cryst. A24, 217 (1968).

[67] I. D. Brown, The Chemical Bond in Inorganic Chemistry: The Bond Valence Model (Oxford University Press, Oxford, 2002).

[68] M. Angst, P. Khalifah, R. Hermann, H. J. Xiang, M. H. Whangbo, V. Varadarajan, J.W. Brill, B.C. Sales, and D. Mandrus, Phys. Rev. Lett. 99, 086403 (2007).

[69] C. H. Li, X. Q. Zhang, Z.H. Cheng, and Y. Sun, Appl. Phys. Lett. 93, 152103 (2008).

[70] B. Fisher, J. Genossar, L. Patlagan, and G. M. Reisner, J. Appl. Phys. 109, 084111 (2011).

[71] N. Ikeda, M. Kubota, H. Hayakawa, H. Akahama, D. Ohishi, A. Nakanishi, T. Funabiki, Y. Matsuo, N. Kimizuka, T. Kambe, S. Mori, and J. Kano, Ferroelectrics 414, 41 (2011).

[72] S. Cao, J. Li, Z. Wang, H. Tian, Y. Qin, L. Zeng, C. Ma, H. Yang, and J. Li, Sci. Rep. 2, 330 (2012).

[73] C. H. Li, X. Q. Zhang, Z.H. Cheng, and Y. Sun, Appl. Phys. Lett. 92, 182903 (2008).

[74] C. H. Li, F. Wang, Y. Liu, X. Q. Zhang, Z. H. Cheng, and Y. Sun, Phys. Rev. B 79, 172412 (2009).

[75] F. Wang, C. H. Li, T. Zou, Y. Liu, and Y. Sun, J. Phys.: Condens. Matter 22, 496001 (2010).

[76] L. J. Zeng, H. X. Yang, Y. Zhang, H. F. Tian, C. Ma, Y. B. Qin, Y. G. Zhao, and J. Q. Li, Europhys. Lett. 84, 57011 (2008).

[77] S. Cao, J. Li, H. F. Tian, Y. B. Qin, L. J. Zeng, H. X. Yang, and J. Q. Li, Appl. Phys. Lett. 98, 102102 (2011).

[78] S. M. Lawrence, PhD thesis, Curtin University, 2011.

[79] F. Wang, T. Zou, Y. Liu, L. Q. Yan, and Y. Sun, J. Appl. Phys. 111, 033703 (2012).
[80] H. Itoh, K. Itoh, K. Anjyo, H. Nakaya, H. Akahama, D. Ohishi, S. Saito, T. Kambe, S. Ishihara, N. Ikeda, and S. Iwai, J. Luminesc. 133, 149 (2013).

[81] F. M. Vitucci, A. Nucara, C. Mirri, D. Nicoletti, M. Ortolani, U. Schade, and P. Calvani, Phys. Rev. B 84, 153105 (2011).

[82] J. de Groot, PhD thesis, RWTH Aachen University, 2012.

[83] C. Lee, J. Kim, S. W. Cheong, and E. J. Choi, Phys. Rev. B 85, 014303 (2012).

[84] H. J. Xiang and M. H. Whangbo, Phys. Rev. Lett. 98, 246403 (2007).

[85] Z. Popović and S. Satpathy, Phys. Rev. Lett. 88, 197201 (2002).

[86] F. M. Vitucci, A. Nucara, D. Nicoletti, Y. Sun, C. H. Li, J. C. Soret, U. Schade, and P. Calvani, Phys. Rev. B 81, 195121 (2010).

[87] Y. Hou, Y. P. Yao, S. N. Dong, M. L. Teng, X. F. Sun, and X. G. Li, J. Raman Spectr. 42, 1695 (2011).

[88] R. J. McQueeney, J. Ma, S. Chang, J. Q. Yan, M. Hehlen, and F. Trouw, Phys. Rev. Lett. 98, 126402 (2007).

[89] H. J. Xiang, E. J. Kan, S. H. Wei, M. H. Whangbo, and J. Yang, Phys. Rev. B 80, 132408 (2009).

[90] A. Nagano, M. Naka, J. Nasu, and S. Ishihara, Phys. Rev. Lett. 99, 217202 (2007).

[91] M. Naka, A. Nagano, and S. Ishihara, Phys. Rev. B 77, 224441 (2008).

[92] J. Nasu, A. Nagano, M. Naka, and S. Ishihara, Phys. Rev. B 78, 024416 (2008).

[93] T. Watanabe and S. Ishihara, J. Phys. Soc. Jpn. 78, 113702 (2009).

[94] T. Watanabe and S. Ishihara, J. Phys. Soc. Jpn. 79, 114714 (2010).

[95] A. J. Hearmon, D. Prabhakaran, H. Nowell, F. Fabrizi, M. J. Gutmann, and P.G. Radaelli, Phys. Rev. B 85, 014115 (2012).

[96] Y. Murakami, N. Abe, T. Arima, and D. Shindo, Phys. Rev. B 76, 024109 (2007).

[97] Y. Horibe, K. Yoshii, N. Ikeda, and S. Mori, Phys. Rev. B 80, 092104 (2009).

[98] M. Tanaka, J. Akimitsu, Y. Inada, N. Kimizuka, I. Shindo, and K. Siratori, Solid State Comm. 44, 687 (1982).

[99] Y. Nakagawa, M. Inazumi, N. Kimizuka, and K. Siratori, J. Phys. Soc. Jpn. 47, 1369 (1979).

[100] N. Ikeda, R. Mori, S. Mori, and K. Kohn, Ferroelectrics 286, 897 (2003).

[101] Y. Horibe, N. Ikeda, K. Yoshii, and S. Mori, Phys. Rev. B 82, 184119 (2010)

[102] Y. B. Qin, H. X. Yang, Y. Zhang, H. F. Tian, C. Ma, Y. G. Zhao, R. I. Walton, and J. Q. Li, J. Phys.: Condens. Matter 21, 015401 (2009).

[103] H. Yang, Y. Zhang, Y. Qin, C. Ma, H. Tian, and J. Li, Phys. Status Solidi B 247, 870 (2010).

[104] Y. B. Qin, H. X. Yang, L. Wang, H. F. Tian, C. Ma, Y. Li, H. L. Shi, and J. Q. Li, Eur. Phys. J. B 75, 231 (2010).

[105] J. Iida, S. Kakugawa, G. Kido, Y. Nakagawa, S. Takekawa, and N. Kimizuka, Physica B 155, 307 (1989).

[106] F. Wang, J. Kim, Y. J. Kim, and G. D. Gu, Phys. Rev. B 80, 024419 (2009). 
[107] W. Wu, V. Kiryukhin, H. J. Noh, K. T. Ko, J. H. Park, W. Ratcliff, P.A. Sharma, N. Harrison, Y.J. Choi, Y. Horibe, S. Lee, S. Park, H. T. Yi, C. L. Zhang, and S. W. Cheong, Phys. Rev. Lett. 101, 137203 (2008).

[108] M. Phan, N. Frey, M. Angst, J. de Groot, B. Sales, D. Mandrus, and H. Srikanth, Solid State Comm. 150, 341 (2010).

[109] Y. Sun, Y. Liu, F. Ye, S. Chi, Y. Ren, T. Zou, F. Wang, and L. Yan, J. Appl. Phys. 111, 07D902 (2012).

[110] J. Iida, M. Tanaka, Y. Nakagawa, S. Funahashi, N. Kimizuka, and S. Takekawa, J. Phys. Soc. Jpn. 62, 1723 (1993).

[111] J. Bourgeois, G. André, S. Petit, J. Robert, M. Poienar, J. Rouquette, E. Elkaïm, M. Hervieu, A. Maignan, C. Martin, and F. Damay, Phys. Rev. B 86, 024413 (2012).

[112] K. Kuepper, M. Raekers, C. Taubitz, M. Prinz, C. Derks, M. Neumann, A. V. Postnikov, F. M. F. de Groot, C. Piamonteze, D. Prabhakaran, and S. J. Blundell, Phys. Rev. B 80, 220409 (2009).

[113] M. Tanaka, H. Iwasaki, K. Siratori, and I. Shindo, J. Phys. Soc. Jpn. 58, 1433 (1989).

[114] S. Nakamura, H. Kito, and M. Tanaka, J. Alloys Compds. 277, 574 (1998).

[115] T. Maruyama, Y. Murakami, D. Shindo, N. Abe, and T. Arima, Phys. Rev. B 86, 054202 (2012).

[116] H. J. Noh, H. Sung, J. Jeong, J. Jeong, S. B. Kim, J. Y. Kim, J. Y. Kim, and B. K. Cho, Phys. Rev. B 82, 024423 (2010).

[117] Y. Tokura and N. Nagaosa, Science 288, 462 (2000).

[118] K. Yoshii, N. Ikeda, Y. Nishihata, D. Maeda, R. Fukuyama, T. Nagata, J. Kano, T. Kambe, Y. Horibe, and S. Mori, J. Phys. Soc. Jpn. 81, 033704 (2012).

[119] S. R. Bland, PhD thesis, Durham University, 2010.

[120] M. Zeng, J. Liu, Y. Qin, H. Yang, J. Li, and J. Dai, Thin Solid Films 520, 6446 (2012).

[121] R. C. Rai, A. Delmont, A. Sprow, B. Cai, and M.L. Nakarmi, Appl. Phys. Lett. 100, 212904 (2012).

[122] W. Wang, Z. Gai, M. Chi, J. D. Fowlkes, J. Yi, L. Zhu, X. Cheng, D. J. Keavney, P.C. Snijders, T.Z. Ward, J. Shen, and X. Xu, Phys. Rev. B 85, 155411 (2012).

[123] C. M. Brooks, R. Misra, J. A. Mundy, L. A. Zhang, B. S. Holinsworth, K. R. O’Neal, T. Heeg, W. Zander, J. Schubert, J. L. Musfeldt, Z. K. Liu, D. A. Muller, P. Schiffer, and D. G. Schlom, Appl. Phys. Lett. 101, 132907 (2012).

[124] M. S. Senn, J. P. Wright, and J. P. Attfield, Nature 481, 173 (2012).

[125] F. Schrettle, S. Krohns, P. Lunkenheimer, V. A. M. Brabers, and A. Loidl, Phys. Rev. B 83, 195109 (2011).

[126] P. Monceau, Adv. Phys. 61, 325 (2012).

[127] P. Lunkenheimer, J. Müller, S. Krohns, F. Schrettle, A. Loidl, B. Hartmann, R. Rommel, M. de Souza, C. Hotta, J. A. Schlueter, and M. Lang, Nat. Mater. 11, 755 (2012).

[128] K. Sedlmeier, S. Elsässer, D. Neubauer, R. Beyer, D. Wu, T. Ivek, S. Tomić, J. A. Schlueter, and M. Dressel, Phys. Rev. B 86, 245103 (2012). 